\title{
Photoemission by Large Electron Wave Packets Emitted Out the Side of a Relativistic Laser Focus
}

\author{
Eric Flint Cunningham \\ Brigham Young University - Provo
}

Follow this and additional works at: https://scholarsarchive.byu.edu/etd

Part of the Astrophysics and Astronomy Commons, and the Physics Commons

\section{BYU ScholarsArchive Citation}

Cunningham, Eric Flint, "Photoemission by Large Electron Wave Packets Emitted Out the Side of a Relativistic Laser Focus" (2011). Theses and Dissertations. 3054.

https://scholarsarchive.byu.edu/etd/3054

This Thesis is brought to you for free and open access by BYU ScholarsArchive. It has been accepted for inclusion in Theses and Dissertations by an authorized administrator of BYU ScholarsArchive. For more information, please contact scholarsarchive@byu.edu, ellen_amatangelo@byu.edu. 
Photoemission by Large Electron Wave Packets Emitted out the Side

of a Relativistic Laser Focus

Eric F. Cunningham

A thesis submitted to the faculty of

Brigham Young University

in partial fulfillment of the requirements for the degree of

Master of Science

Michael Ware, Chair

Justin Peatross

Scott Bergeson

Department of Physics and Astronomy

Brigham Young University

August 2011

Copyright @ 2011 Eric F. Cunningham

All Rights Reserved 


\author{
ABSTRACT \\ Photoemission by Large Electron Wave Packets Emitted out the Side \\ of a Relativistic Laser Focus \\ Eric F. Cunningham \\ Department of Physics and Astronomy \\ Master of Science
}

There are at least two common models for calculating the photoemission of accelerated electrons. The 'extended-charge-distribution' method uses the quantum probability current (multiplied by the electron charge) as a source current for Maxwell's equations. The 'point-like-emitter' method treats the electron like a point particle instead of like a diffuse body of charge. Our goal is to differentiate between these two viewpoints empirically.

To do this, we consider a large electron wave packet in a high-intensity laser field, in which case the two viewpoints predict measurable photoemission rates that differ by orders of magnitude. Under the treatment of the 'extended-charge-distribution' model, the strength of the radiated field is significantly limited by interferences between different portions of the oscillating charge density. Alternatively, no suppression of photoemission occurs under the 'point-like-emitter' model because the electron is depicted as having no spatial extent.

We designed an experiment to characterize the photoemission rates of electrons accelerated in a relativistic laser focus. Free electron wave packets are produced through ionization by an intense laser pulse at the center of a large vacuum chamber. These quantum wave packets can become comparable in size to the laser wavelength through natural spreading and interactions with the sharp ponderomotive gradients of the laser focus. Electron radiation emitted out the side of the focus is collected by one-to-one imaging into a 105-micron gold-jacketed fiber, which carries the light to a single photon detector located outside the chamber. The electron radiation is red-shifted due to mild relativistic acceleration, and we use this signature to spectrally filter the outgoing light to discriminate against background. In addition, the temporal resolution of the electronics allows distinction between light that travels directly from the focus into the collection system and laser light that may scatter from the chamber wall.

Keywords: single electron radiation, large wave packet, high-intensity laser, photoemission, scattering, quantum optics, experimental physics 


\section{ACKNOWLEDGMENTS}

I would like to acknowledge the financial support of the National Science Foundation, as well as the financial and academic support of Brigham Young University. I also acknowledge the hard work, leadership, and guidance of Dr. Ware, Dr. Peatross, and Dr. Bergeson. I am highly appreciative of the camaraderie and support shown by John Corson and Nathan Heilmann, our group's other graduate students. I also acknowledge the helpful assistance of Colin Mann, Jacob Johansen, Kamal Pangeni, Nick Herrick, Phillip Smith, Brian Crenshaw, and Grayson Tarbox in various as-

pects of this experiment. I also acknowledge the support of my family, especially from my wife, Emma Cunningham. 



\section{Contents}

Table of Contents $\quad$ v

$\begin{array}{ll}\text { List of Figures } & \text { vii }\end{array}$

1 Introduction $\quad 1$

1.1 Free Electron Wave Packets in a Laser Field . . . . . . . . . . . . . . . . . 1

1.2 Related Work from Literature . . . . . . . . . . . . . . . . . . . 3

1.3 Motivation for Experiment ....................... 5

1.3.1 Classical Point Charge in a High-Intensity Focus . . . . . . . . . . . 5

1.3.2 Extended Charge Distribution in an External Field . . . . . . . . . . . 7

1.4 Summary of Results . . . . . . . . . . . . . . . . . . . 10

2 Experimental Setup $\quad 11$

2.1 Overview of Experiment . . . . . . . . . . . . . . . . 11

2.2 Laser System . . . . . . . . . . . . . . . . . . . . . . . 13

2.3 Compression Chamber . . . . . . . . . . . . . . . . . . . . . . . . . . . . . .

2.4 High-Intensity Focus . . . . . . . . . . . . . . . . . . . 18

2.5 Vacuum and Gas Systems . . . . . . . . . . . . . . . . . . . 23

2.6 Collection of Electron Radiation . . . . . . . . . . . . . . . . . . . 25

2.7 Temporal Filtering . . . . . . . . . . . . . . . . 26

2.8 Efficient, Light-Tight Optical Fiber ～. . . . . . . . . . . . . . . . . . 27

2.9 Spectral Filtering . . . . . . . . . . . . . . . . . . . . . . . . . . . . . . . . . . . . . . . .

2.10 Detection System . . . . . . . . . . . . . . . . . . . 29

3 Experimental Results

3.1 Effect of Spectral Filtering . . . . . . . . . . . . . . . . . . 31

3.2 Comparison to Predicted Red-Shifted Photon Count . . . . . . . . . . . . . . 35

3.3 Conclusions . . . . . . . . . . . . . . . . . . 36

3.4 Future Work . . . . . . . . . . . . . . . . . . . 36

3.4.1 Preparation for Electron Radiation Experiment . . . . . . . . . . . . . 36

3.4 Parametric Down-Conversion . . . . . . . . . . . . . . . . 37

3.4.3 Spectral Make-up of Background . . . . . . . . . . . . . . . . 38 
3.4.4 Signal Strength and Interpretation Issues $\ldots \ldots . \ldots . \ldots$

A Scattering by a Current Distribution 41

B Ionization Volume of a Laser Focus 45

$\begin{array}{ll}\text { Bibliography } & 51\end{array}$

$\begin{array}{ll}\text { Index } & 55\end{array}$ 


\section{List of Figures}

1.1 Cross-section of large electron wave packet in strong laser field . . . . . . . . . . 4

1.2 Classical electron in laser focus: trajectory and radiation . . . . . . . . . . 6

1.3 Red-shifted photoemission from classical electron in laser focus . . . . . . . . . 7

1.4 Photoemission from various sizes of classical extended current distributions . . . . 8

1.5 Efficiency of far-field light scattering as a function of size of the charge distribution 9

2.1 Overall schematic for electron radiation experiment . . . . . . . . . . . . . 12

2.2 The beam path from the laser system to the compression chamber $\ldots \ldots \ldots$

2.3 Beam path into the compression chamber . . . . . . . . . . . . . . . 14

2.4 Live (Raw) FROG showing pulse compression . . . . . . . . . . . . . . . 15

2.5 Beam path inside the compression chamber . . . . . . . . . . . 16

2.6 Laser focus at the pinhole . . . . . . . . . . . . . . . 17

2.7 Time-of-flight spectrometer: setup . . . . . . . . . . . . . . . . . . 19

2.8 Time-of-flight intensity measurement: $\mathrm{Ar}^{+}$through $\mathrm{Ar}^{8+} \ldots \ldots \ldots \ldots$

2.9 Time-of-flight histogram: $\mathrm{Ar}^{9+}$ and $\mathrm{Ar}^{10+} \ldots \ldots \ldots \ldots . \ldots . \ldots 22$

2.10 Time-of-flight histogram comparison: argon vs. vacuum . . . . . . . . . . 22

2.11 Setup for introducing gas into the interaction chamber . . . . . . . . . . . . . 24

2.12 Collection of electron radiation . . . . . . . . . . . . . . 25

2.13 Arrival time of laser pulse . . . . . . . . . . . . . . . . . 26 
2.14 Setup for spectral filtering and photon detection . . . . . . . . . . . . 27

2.15 Manufacturer's transmission specifications of spectral filters $\ldots \ldots$. . . . . . 28

2.16 Schematic for spectral filter box . . . . . . . . . . . . . . . . 29

2.17 Manufacturer's specification of photon detection efficiency of avalanche photodiode 30

3.1 Example of reconstruction of noise background using neutral-density filters . . . 32

3.2 Effect of spectral filtering on noise background . . . . . . . . . . . 33

3.3 Effect of spectral filtering on noise background (elliptical mirror) . . . . . . . . 35

3.4 Schematic for down-conversion setup . . . . . . . . . . . . . . 37

B.1 Laser focus dogbone . . . . . . . . . . . . . . . . 45

B.2 Scaled ionization focal volume of argon ions: $\mathrm{Ar}^{+}$to $\mathrm{Ar}^{8+} \ldots \ldots . \ldots$

B.3 Scaled ionization focal volume of argon ions: $\mathrm{Ar}^{9+}$ and $\mathrm{Ar}^{10+} \ldots \ldots$. . . . . . 49 


\section{List of Tables}

2.1 Time-of-flight time delay and corresponding ionization intensity for argon . . . . 20 


\section{Chapter 1}

\section{Introduction}

\subsection{Free Electron Wave Packets in a Laser Field}

A charged particle emits electromagnetic radiation when accelerated by a laser field. In classical electrodynamics, this photoemission is calculated while treating the electron as a point charge. When considering the description of particles in quantum mechanics, however, the electron evolves as an extended wave function instead. These two descriptions have led to different ideas of how one should compute the radiation of laser-driven electrons.

Intuitively, one might expect to account for quantum mechanics by treating the wave packet like a diffuse, classical extended charge distribution [1,2]. In this viewpoint - held even by Schrödinger himself — the evolution of the wave packet is governed by quantum mechanics, while its photoemission is still determined using classical electrodynamics [3].

As long as the wave packet remains small compared to the wavelength of the driving laser, the diffuse nature of the electron wave packet does not induce an appreciable departure from the classical behavior of a point particle under the 'extended-charge-distribution' model described above. However, if the wave packet grows larger, its dynamics become highly nonclassical: As the 
spatial extent of the electron wave packet becomes comparable to the laser wavelength, different parts of the wave packet experience different phases of the laser field and oscillate out of phase with respect to each other.

Investigating these dynamics, Chowdhury et al. [1] used the 'extended-charge-distribution' method to describe the radiation from a large electron wave packet in a strong laser field. Because of the phase mis-match between oscillating portions of the extended charge distribution, the classically-calculated photoemission exhibited self-interference. This led to an order-of-magnitude difference in the predicted Larmor radiation yield compared to the radiation from a laser-driven point electron.

These results present some conceptual difficulties associated with calculating radiation under the 'extended-charge-distribution' model. The suppression of radiation in this model depends on having one part of the wave packet (charge distribution) perform work on another part of the wave packet, even though the entire wave packet represents a single electron. Furthermore, a complete description of the radiative process for a large wave packet accelerated in an electric field requires a quantum mechanical description of both the electron and the emitted light. It is not obvious that such a description would reduce to the 'extended-charge-distribution' model.

A more accurate model of this system interprets an extended wave packet instead as the possible location of a point-like emitter, with the various possibilities doing no work on one another. Some theoretical work by Peatross et al. invokes the framework of quantum electrodynamics to support this 'point-like-emitter' viewpoint [4]. John Corson recently extended the theoretical analysis, showing that an electron wave packet does no work on itself. Treating both the electron and the photemission using a quantum-electrodynamical approach, his work demonstrates that electrons radiate similar to point-like particles [5].

The theoretical work also suggests an experimental difference between the two approaches [4] (discussed later in Section 1.3). In this thesis, we report our design for such an experiment, in 
which we measure the photoemission rates of large electron wave packets in a high-intensity laser focus. In addition, we detail our progress towards a definitive measurement.

\subsection{Related Work from Literature}

The investigation into dynamics of an electron wave packet evolving in a super-intense laser field has received considerable attention in the field of laser-matter interactions over the past decade $[1,2,4-16]$. In this section, we review some literature relevant to our experiment that examines these dynamics.

In 2000, San Roman et al. [6] constructed a laser-driven wave packet representing a free Dirac electron through an analytically-determined superposition of Volkov solutions to the Dirac equation. In carrying out the numerical simulation using ultraviolet laser frequencies and a stronglyrelativistic intensity $\left(3 \times 10^{21} \mathrm{~W} / \mathrm{cm}^{2}\right)$, the authors identified various electron dynamics, including the squeezing and drifting of wave packets due to the strong magnetic field of the laser.

The same group furthered their work by exploring an electron wave packet's interaction with a strong, short pulse in 2001 [7]. They investigated the evolution of Gaussian wave packets under the influence of $800 \mathrm{~nm}$ titanium-sapphire pulses, considering both the small-packet and largepacket regimes. The result of the small-packet (width of $1.25 \mathrm{~nm}$ ) simulation closely resembles the evolution of an ensemble distribution of individually-simulated classical point charges. In comparison, the large-packet (width of $278.5 \mathrm{~nm}$ ) simulation exhibits dramatically nonclassical dynamics, including a "double hump" structure at the classical turning points.

Unfortunately, these calculations of electron dynamics proved difficult and time-consuming due to the four-component nature of the Dirac equation. In 2002, however, Panek et al. [8] considered nonlinear Compton scattering under both the spinless Klein-Gordon equation and the fulltreatment Dirac equation. By comparing the results, they showed that spin effects at high intensities 
are typically minimal. Work by Walser et al. in 2002 [9] and Mocken et al. in 2003 [10] reached the same consensus in other areas of investigation.

These findings motivated the use of the Klein-Gordon equation for simulating free-electron dynamics rather than the computationally-challenging Dirac equation. In 2007, Peatross et al. [11] implemented various expansions and approximations under this more-manageable framework. These simplifications allowed for the construction of a wave packet analytically from Volkovstate solutions to the Klein-Gordon equation in a relativistic electromagnetic field. As a result, the authors substantially reduced numerical demands on computational power. This allowed them to animate two-dimensional cuts through a three-dimensional wave packet evolving in an electromagnetic field, using only a basic laptop computer. Many non-dipole effects were clearly visible, including the previously-identified packet-squeezing, Lorentz drift, and "double hump" [6,7]. The spatial extent of these wave packets was also shown to grow to sizes comparable to the laser wavelength, as shown in Fig. 1.1. This stirred interest in the question of how such packets radiate.

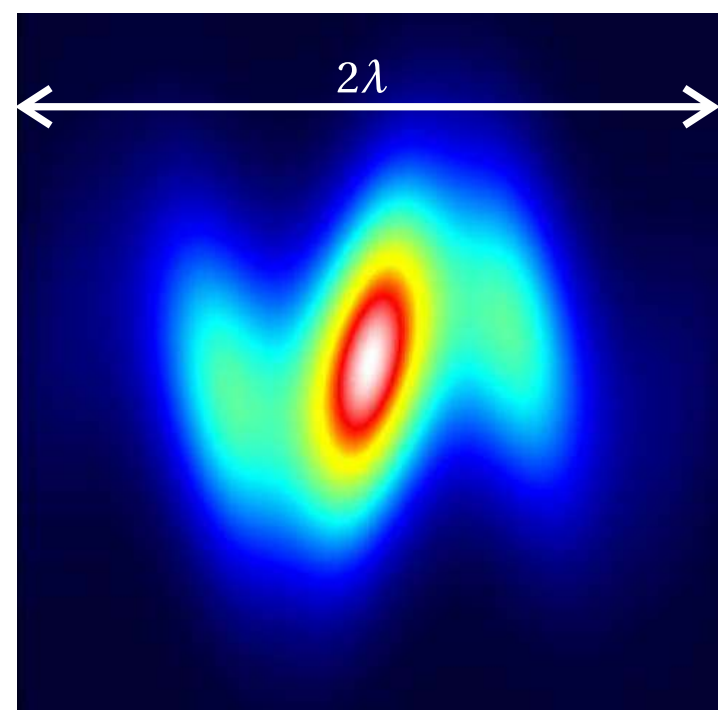

Figure 1.1 Large electron wave packet after spreading for 125 cycles in a strong laser field. The wave packet was constructed using Volkov-state solutions to the Klein-Gordon equation [11]. 


\subsection{Motivation for Experiment}

In a subsequent study, Peatross et al. [4] investigated the photoemission from large electron wave packets in a strong laser field. In addition to a quantum-electrodynamics analysis, the authors calculated the expected photoemission from a point charge accelerated in a high-intensity laser focus (Section 1.3.1) and from an extended body of charge in an external field (Section 1.3.2). By comparing the two predictions, they found there should be an experimental difference between the photoemission of large electron wave packets under the 'extended-charge-distribution' model and the 'point-like emitter' model [4]. As this study is the origin of the idea for our experiment, we review the authors' findings below.

\subsubsection{Classical Point Charge in a High-Intensity Focus}

In the presence of a high-intensity, linearly-polarized laser, a classical point particle oscillates in the plane of the electric field. Because of the high intensity, the magnetic field of the laser becomes significant, and the electron also drifts downstream due to the Lorentz force. Figure 1.2 shows this type of trajectory: beginning on-axis in the laser focus, the electron generally oscillates in the $\mathrm{x}$-dimension and drifts in the z-dimension. Eventually, the electron shoots out the side of the focus due to the strong ponderomotive gradient. The trajectory is calculated by approximating the full four-vector potential inside the focus and allowing a particle to evolve in time according to the relativistic Lorentz force.

Using the particle trajectory, Peatross et al. calculated the far-field Larmor radiation [3] using parameters appropriate for the laser focus in an experiment (wavelength of $800 \mathrm{~nm}$, pulse duration

of $35 \mathrm{fs}$, peak intensity of $10^{19} \mathrm{~W} / \mathrm{cm}^{2}$, beam waist of $\left.2.4 \mu \mathrm{m}\right)$. This system yielded a total radiated energy of $0.24 \mathrm{eV}$ into all solid angles [4]. The classical trajectory and its total radiation pattern are shown in Fig. 1.2. 

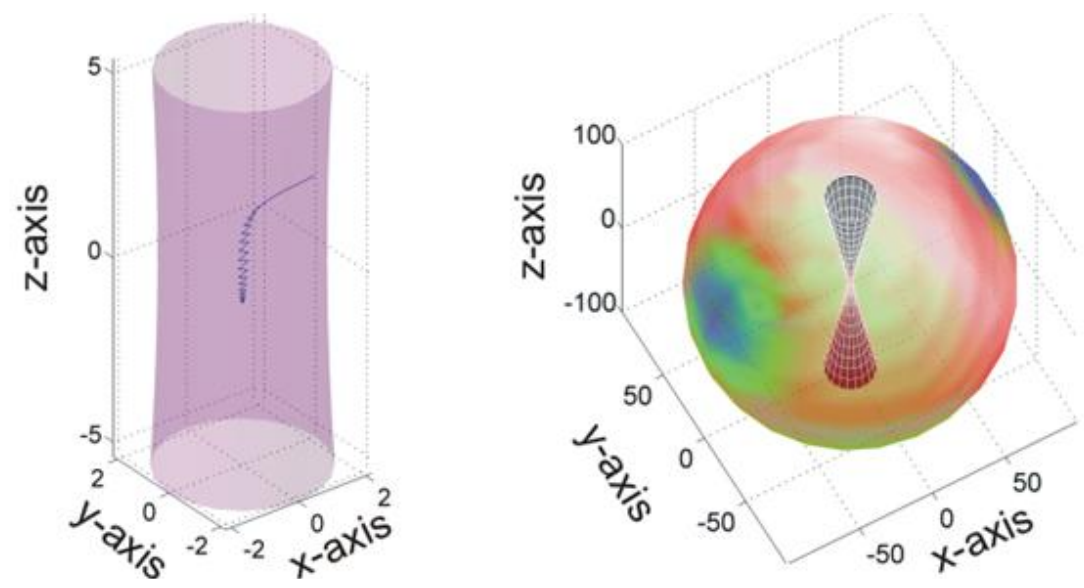

Figure 1.2 Trajectory of a classical electron in a laser focus (left) with its associated radiation pattern (right) [4].

Unsurprisingly, this photoemission hardly compares in magnitude to the light from the electron's driving laser, making it seemingly impossible to separate electron radiation from laser radiation in an experiment. Consider that if the average energy radiated in all directions is only $0.24 \mathrm{eV}$, and if we place a detector where $20 \%$ of that energy could be collected, then the average photoemission energy detectable from each shot is even smaller:

$$
\left(\frac{0.24 \mathrm{eV} \text { total }}{\text { shot }}\right)(0.2 \text { collected })=\frac{0.048 \mathrm{eV} \text { collected }}{\text { shot }}
$$

Comparing with the laser photon energy of $1.55 \mathrm{eV}$, this would mean about 1 photon per 32 laser shots. In contrast, a single $40 \mathrm{~mJ}$ driving laser pulse from a titanium-sapphire laser has about $10^{17}$ photons. The two photon amounts differ by over eighteen orders of magnitude!

This comparison highlights the significance of the Doppler shift to our experiment. As the point particle in Fig. 1.2 accelerates forward due to the Lorentz force, its velocities attain levels significant compared to the speed of light. This relativistic motion causes a red-shift in the photoemission perpendicular to the plane of the laser polarization, as observed out the lateral side of the focus. Figure 1.3 shows the radiation pattern for light in the $850 \mathrm{~nm}$ to $950 \mathrm{~nm}$ range, which accounts for $20 \%$ of the total emitted power [4]. 


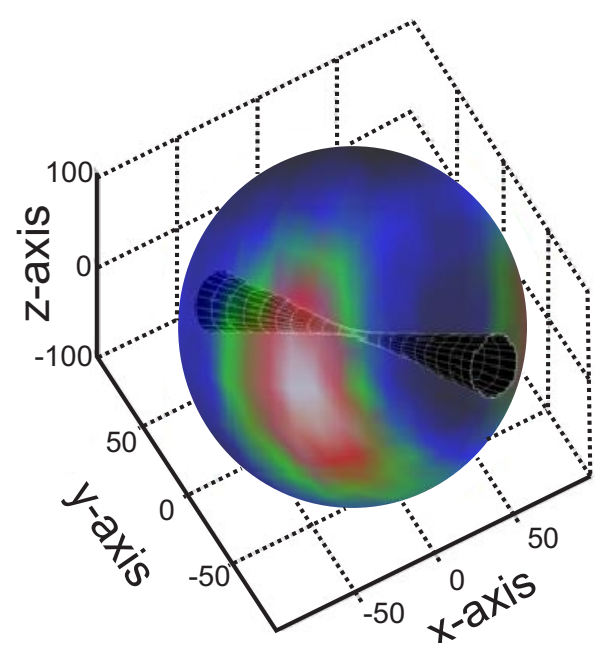

Figure 1.3 Red-shifted photoemission of the classical electron in a laser focus, whose trajectory is shown in Fig. 1.2 [4].

Because of the difference in wavelength, we can distinguish electron photoemission chromatically from the non-red-shifted photons of the laser in an experiment using frequency-dependent filters. Despite an even lower predicted count rate of 1 photon every 300 shots into the solid angle of the collection lenses [4], the spectral signature allows us to isolate a single-photon signal over many thousands of shots, even in the midst of the $10^{17}$ photons of each laser pulse.

\subsubsection{Extended Charge Distribution in an External Field}

The red-shift in radiation provides evidence that photoemission from a laser-driven point charge could be measured experimentally. However, this possibility only matters if one can establish a testable difference between photoemission under the 'point-like-emitter' model and the 'extendedcharge-distribution' model. To this end, Peatross et al. compared the photoemission in Fig. 1.2 to the radiation pattern derived from an extended charge distribution in an external field. To model this behavior, the authors considered a system of a Gaussian current distribution driven by a plane wave in the low-intensity limit [4]. This is given by

$$
\vec{J} \propto \hat{z} r_{0}^{-3} e^{-r^{2} / r_{0}^{2}} e^{i(k x-\omega t)}
$$


It is shown in Appendix A that this current distribution leads to a Thomson scattering intensity distribution of

$$
I(\theta, \phi) \propto \sin ^{2} \theta e^{-k^{2} r_{0}^{2}(1-\sin \theta \cos \phi)}
$$

Examine the exponent in the intensity distribution. As the current distribution size $r_{0}$ grows, the photoemission pattern changes significantly due to destructive interference. As the size of the current distribution begins to rival the wavelength of the driving plane wave, the photoemission quickly becomes limited to the forward direction only, as shown in Fig. 1.4 [4].

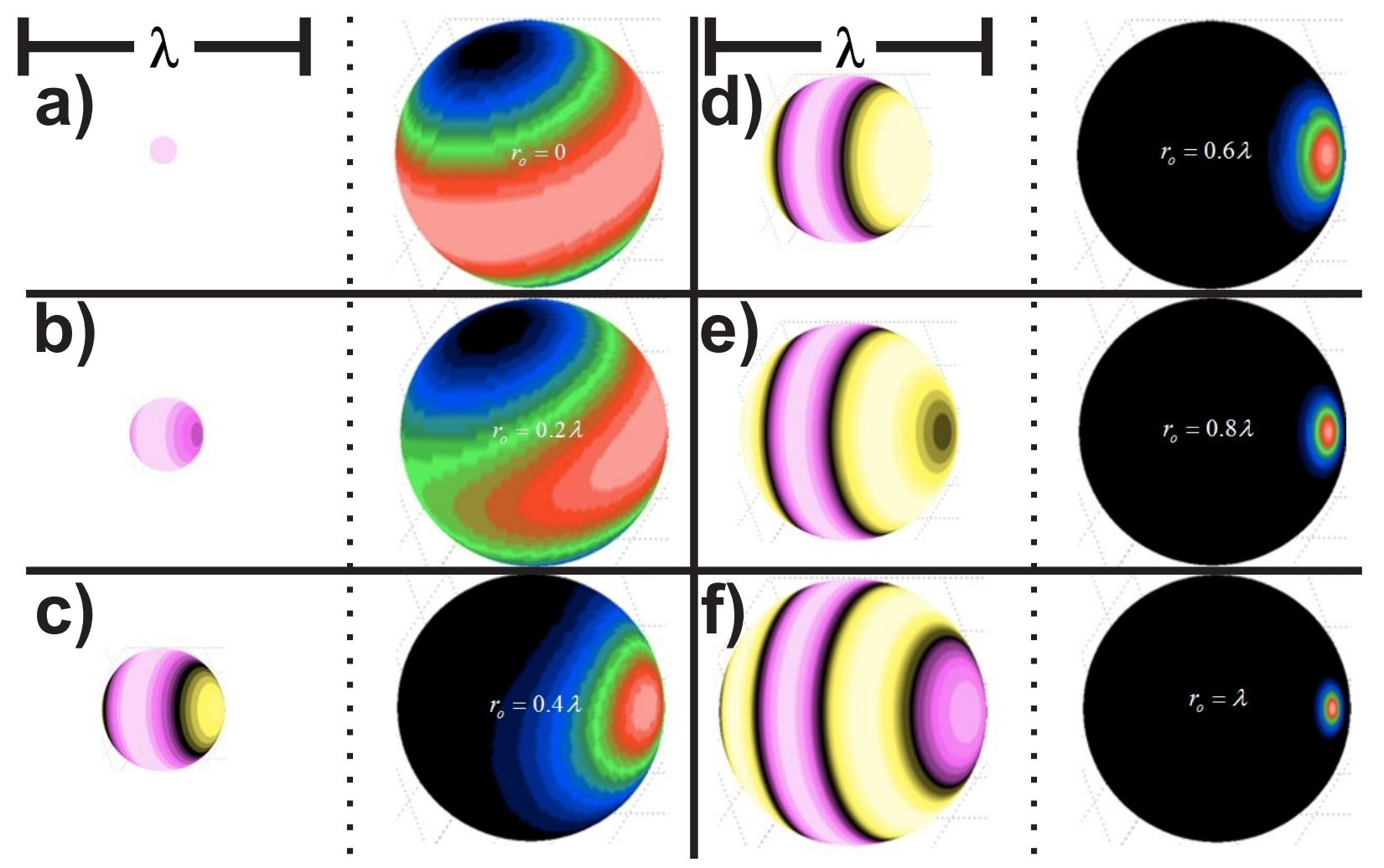

Figure 1.4 Photoemission from various sizes of classical extended current distributions driven by a plane wave in the low-intensity limit, increasing evenly from $r_{0}=0$ to $r_{0}=\lambda$. Each distribution is shown to the left of its corresponding intensity pattern.

We display the effects of the current distribution size on the far-field scattering efficiency in Fig. 1.5. When the size of the current distribution is small, the light scatters equally well in all directions. As the size increases, however, only light in the forward direction continues to scatter 
coherently. This results in a sharp drop-off in scattering efficiency in the perpendicular and reverse directions, especially as the current distribution size approaches and surpasses the scale of the wavelength of the driving plane wave.

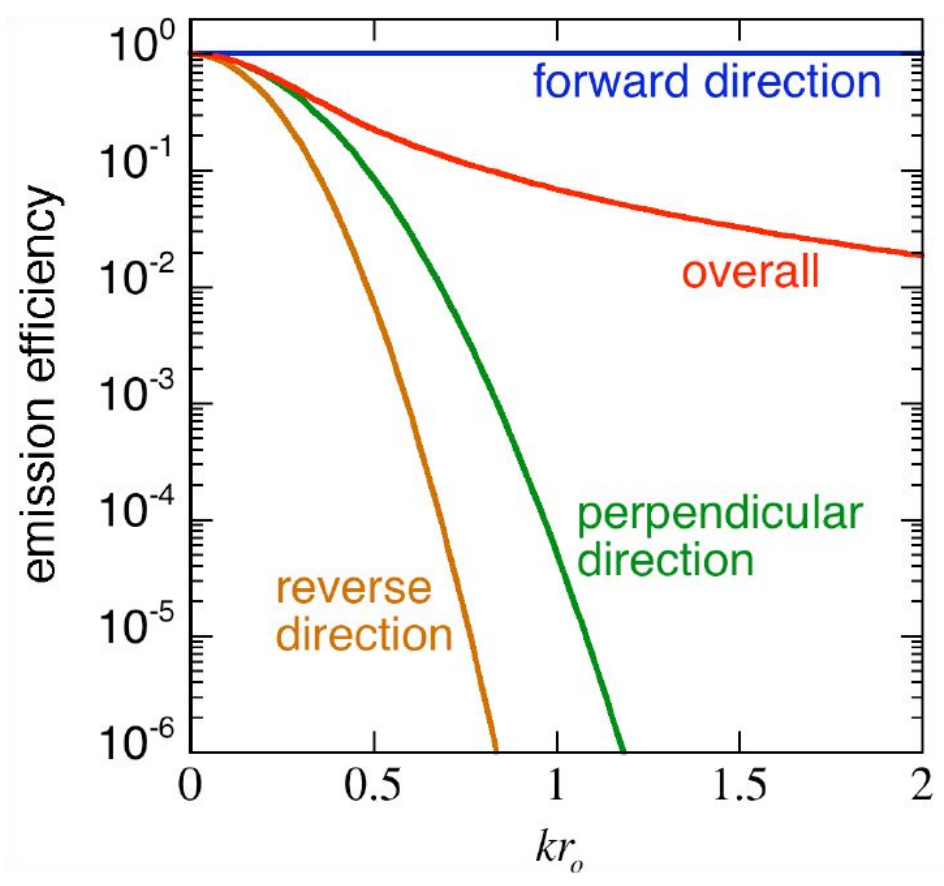

Figure 1.5 Efficiency of far-field light scattering as a function of the size of the current distribution [4].

Note in Fig. 1.4 and Fig. 1.5 that a large-scale charge distribution in an external field does not radiate well in the lateral direction. In comparison, the driven point charge in Fig. 1.2 and Fig. 1.3 radiates much more efficiently in this direction. By contrasting the radiation patterns in these figures, Peatross et al. recognized a difference between the photon count rates of two orders of magnitude in the lateral direction [4]. Coincidentally, this is the same direction in which radiated photons can be distinguished from laser photons due to the red-shift in wavelength. This combination provides the opportunity for experimental differentiation between the two models. 


\subsection{Summary of Results}

We have designed and constructed an experiment to test how an electron radiates when its wave packet reaches spatial scales comparable to the wavelength of the accelerating electromagnetic field. In comparing the theoretical photoemission rates from the 'point-like-emitter' method to the 'extended-charge-distribution' method, the predicted number of photons emitted out the side of the relativistic laser focus differs by two orders of magnitude (see Fig. 1.2 and Fig. 1.4). Because we can also discriminate radiated photons spectrally from laser photons in this direction, the number of red-shifted photons detected out the lateral side of a relativistic laser focus provides an observable indicator as to whether large electron wave packets radiate like point-like emitters or like extended charge distributions.

With our laser system and elliptical mirror, we were able to achieve intensities in excess of $2 \times 10^{18} \mathrm{~W} / \mathrm{cm}^{2}$, which is strong enough to accelerate the electrons to mildly-relativistic speeds. Using spectral filters, we reduced the noise background during the time interval of interest by about six orders of magnitude. As a result, the predicted red-shifted photon count rate in the lateral direction is bigger than this filtered noise background by nearly a factor of ten. 


\section{Chapter 2}

\section{Experimental Setup}

In this chapter, we detail the assembly and calibration of the experimental apparatus. After providing a general overview of the experiment, we add details concerning the laser system, pulse compression, high-intensity focus, vacuum system, collection and fiber system, filtering systems, and detection system.

\subsection{Overview of Experiment}

Our experiment is designed to measure photons emitted laterally from large electron wave packets in a high-intensity laser focus. These photons have characteristic temporal and spectral features, allowing us to distinguish them from the background (see Section 1.3.1). The goal is to differentiate between 'point-like' and 'extended-charge-distribution' emission of single electrons in a scenario when the size of the electron wave packet is larger than the laser wavelength.

The general layout of the experiment is shown in Fig. 2.1. We use the titanium-sapphire laser (top-left corner of Fig. 2.1) in the lab of Dr. Peatross as the high-intensity light-source in the photon measurements. The infrared pulses of the laser system are stretched and amplified before propagating into the lab of Dr. Ware, where the pulses enter the first of two vacuum chambers. 


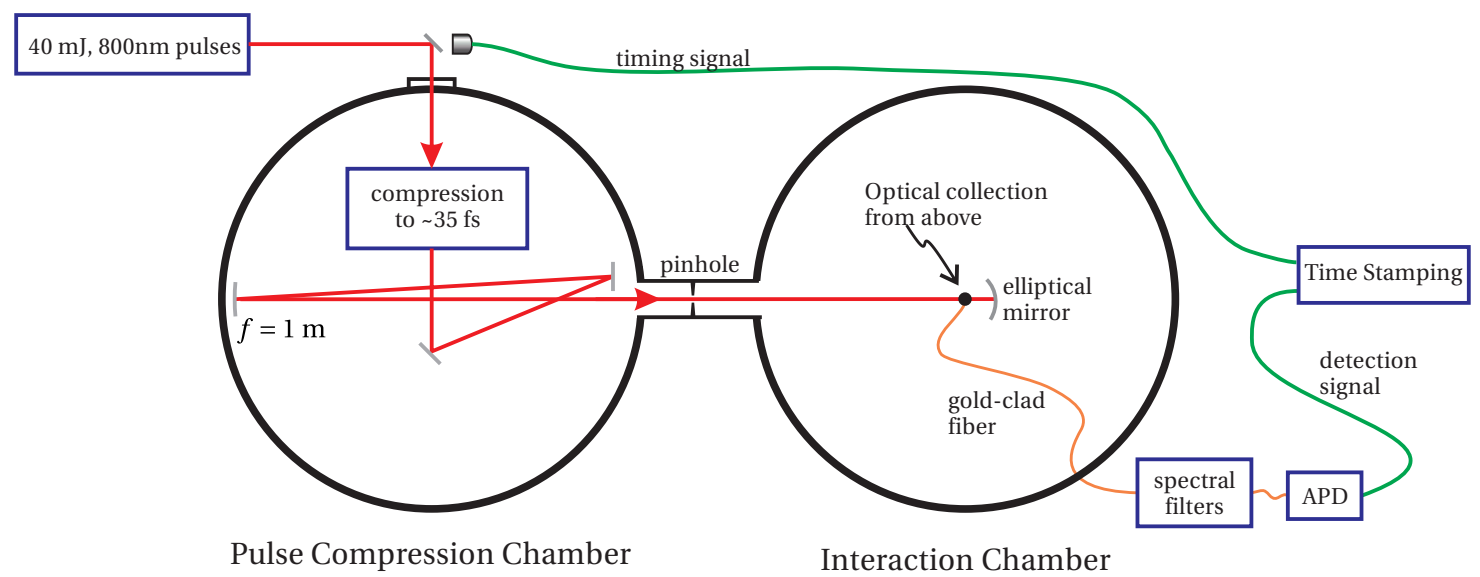

Figure 2.1 Schematic showing the overall layout of the electron radiation experiment.

Inside this chamber (left side of Fig. 2.1), the pulses are temporally compressed to 35 fs using a diffraction grating setup. These compressed pulses are then directed out of the compression chamber, through a pinhole, and into the interaction chamber (right side of Fig. 2.1).

In the electron radiation experiment, the interaction chamber is pumped down to high vacuum $\left(<10^{-8}\right.$ torr) and then back-filled to $10^{-6}$ torr with helium. An elliptical mirror reflects the incoming pulse from the pinhole, and the focused laser achieves a peak intensity in excess of $10^{18} \mathrm{~W} / \mathrm{cm}^{2}$. On average, only a few individual atoms are located in this region of highest intensity, and each one is doubly ionized by the leading edge of the laser pulse. The free electron wave packets spread and react to the strong ponderomotive gradients in the laser focus. The high intensity also provides a relativistic Lorentz drift in the direction of the laser propagation, accelerating the electrons to a significant fraction of the speed of light. This causes the photons emitted from the area of highest intensity to be red-shifted when viewed from the side of the laser focus. This red-shift provides a characteristic frequency signature allowing us to differentiate between the photoemission of the relativistic electrons and the background laser noise.

Above the laser focus, light is collected into a gold-coated optical fiber, which guides the signal into a system of spectral filters (bottom-right corner of Fig. 2.1). These filters greatly suppress the 
fundamental laser wavelength while transmitting about $70 \%$ of the red-shifted photons into another fiber. The light finally enters an avalanche photodiode capable of detecting these individual redshifted photons with a $35 \%$ efficiency. The arrival of each photon is time-stamped and compared to the trigger from the laser by a time digitizer. Because the interaction chamber is large, the difference between light collected from reflections off the chamber wall and light collected directly from the focus is distinguishable using timing.

\subsection{Laser System}

Our experiment requires high intensities in order to accelerate the target electrons to relativistic speeds. We use an existing high-intensity titanium-sapphire (Ti:S) laser system, capable of achieving $100 \mathrm{~mJ}$ pulses compressed to a temporal duration of $35 \mathrm{fs}$ with a repetition rate of $10 \mathrm{~Hz}$. After amplification, the pulse in the Peatross lab is directed away from the laser system, through a shaft in the wall, and into the Ware lab, where an optics table supports the two vacuum chambers. The diagram in Fig. 2.2 represents the general layout of this setup.

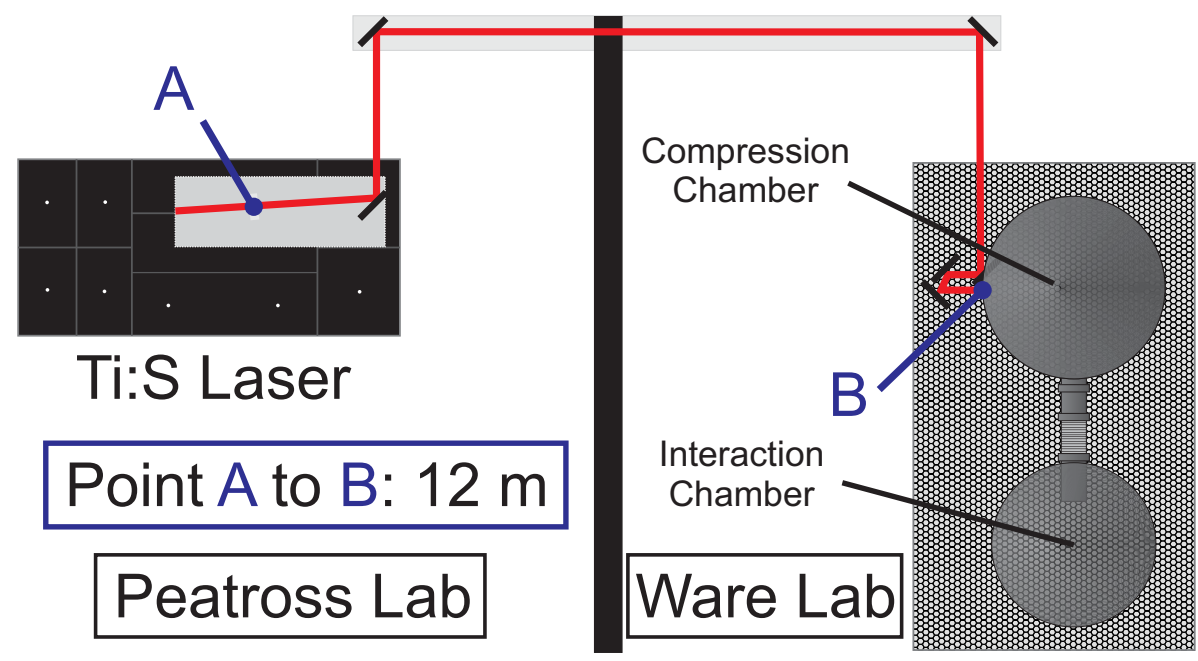

Figure 2.2 The beam path from the laser system in the Peatross lab to the compression chamber in the Ware lab. 
To properly compress the pulse, the laser must be collimated before passing through the diffraction grating pair. One common technique for collimation uses a telescope, which collimates the laser and expands the beam to prevent damage to the gratings. We elected not to use this method because of aberration introduced into the beam by the extra lenses. Furthermore, beam expansion is unnecessary because the diverging pulse propagates twelve meters before reaching the diffraction gratings. By then, the beam diameter enlarges enough that it does not harm the gratings.

Instead of a telescope, we use a twelve meter-focal length lens as the window of the vacuum chamber. This focal length matches the distance to the final concave lens in the laser system from whence the beam diverges (as shown in Fig. 2.2). This effectively collimates the beam in preparation for pulse compression. Figure 2.3 shows the path of the laser into the chamber.

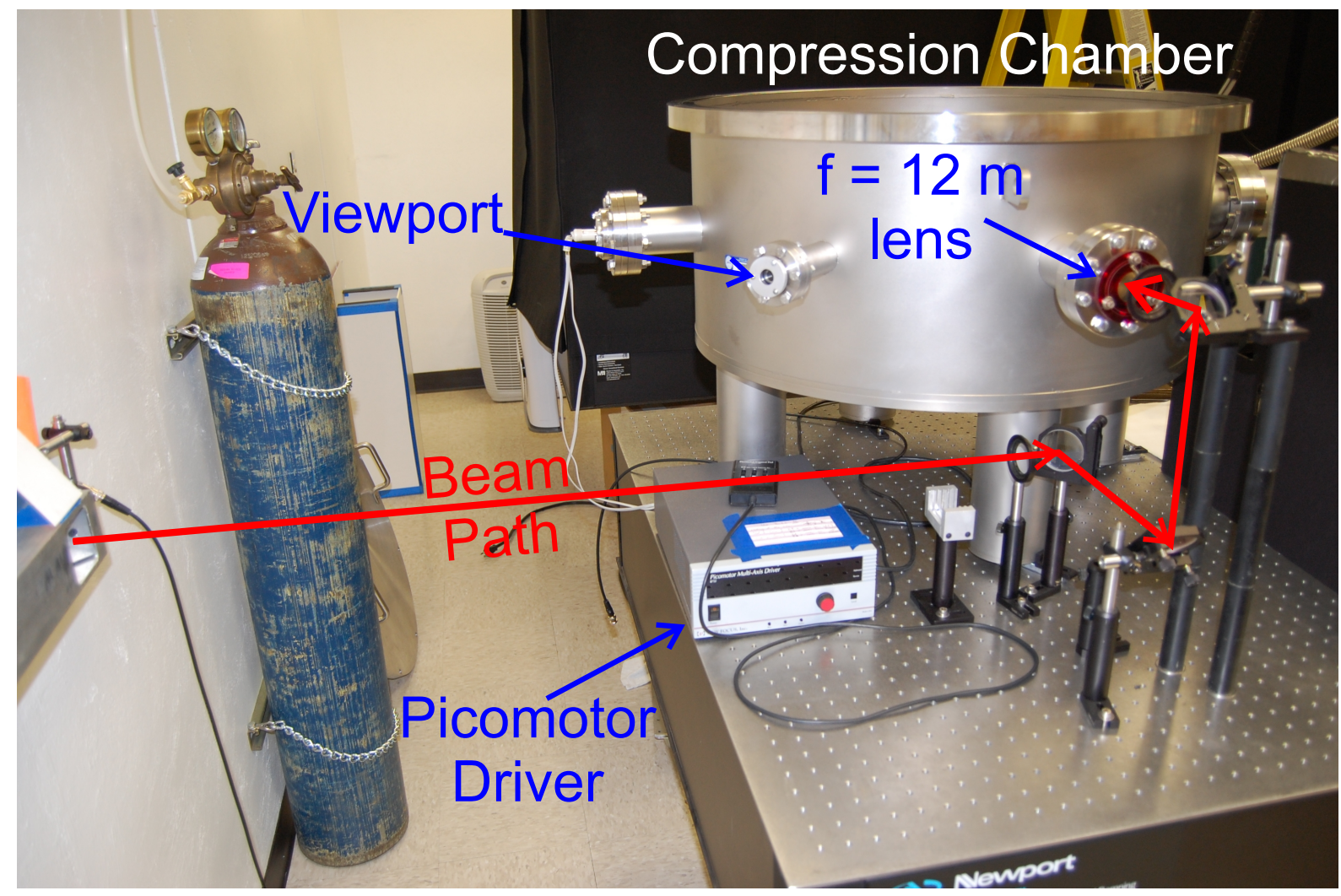

Figure 2.3 Path of the incoming laser pulse introduced from the shaft on the left and entering the compression chamber on the right. 


\subsection{Compression Chamber}

The collimated pulse enters the compression chamber and immediately encounters the first of two parallel diffraction gratings, causing the laser's different frequencies to spread out spatially. The gratings are situated in such a way that the laser pulse's main wavelengths reflect off both gratings, while any unwanted $900 \mathrm{~nm}$ light from the laser amplifiers misses the second grating entirely. (This serves as an important noise control since the red-shifted electron radiation is expected to fall in this wavelength range.) The pulse is then sent back through the grating pair to undo the spatial chirp. (Refer later to Fig. 2.5 for the complete beam path throughout the compression chamber.)

We optimized the grating spacing by aiming the unfocused, compressed beam into a Swamp Optics GRENOUILLE instrument controlled by a VideoFROG computer program. While modifying the gratings' positioning, we viewed the GRENOUILLE's pulse duration measurements on a computer screen in real-time. This allowed us to see the change in the pulse's temporal compression after each adjustment. We continued this process until the pulses regularly achieved a temporal duration of 35 fs. Figure 2.4 shows a typical VideoFROG trace of one of the laser pulses.

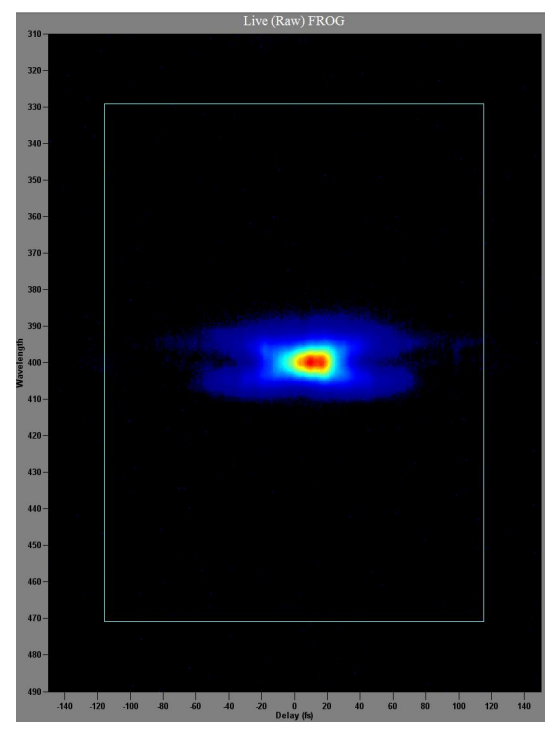

Figure 2.4 VideoFROG trace of a pulse compressed to $37 \mathrm{fs}$ as measured using frequency-resolved optical gating. 
After compression, the pulse is reflected off Mirror 1 (as labeled in Fig. 2.5), which is directly below the pulse's incoming position. The pulse is then directed off Mirror 2 to Mirror 3 located at the mouth of a deep exit port leading to the interaction chamber. This mirror is surrounded on the back side with aluminum foil to prevent scattered light from entering the interaction chamber ahead of the main laser pulse.

Mirror 3 directs the pulse at constant height to the center of a one-meter-focus spherical mirror, which is aligned exactly opposite from a pinhole centered at the far inside end of the exit port. The spherical mirror focuses the laser through the pinhole into the interaction chamber. The red arrows in Fig. 2.5 denote the path of the laser pulse throughout the compression chamber.

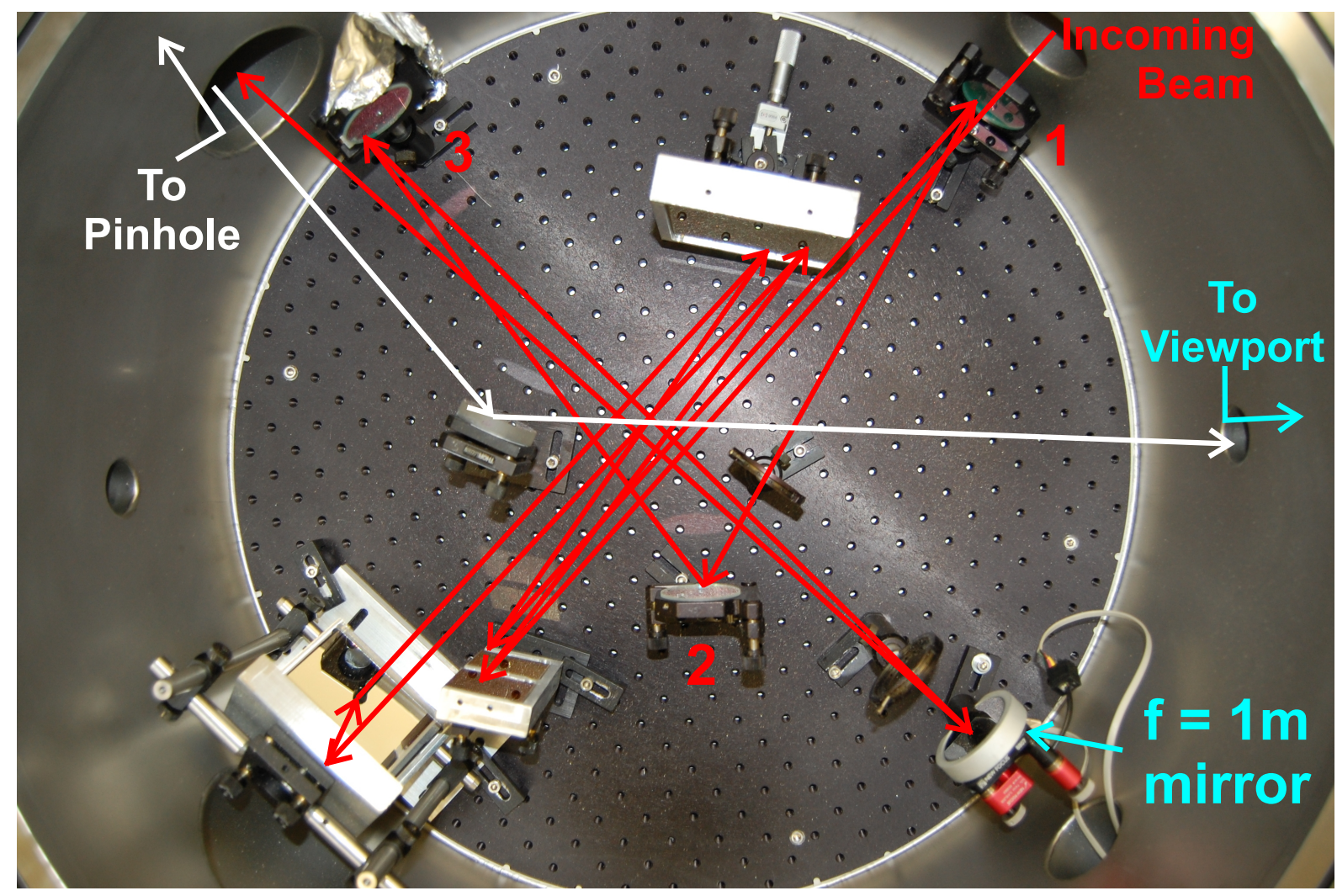

Figure 2.5 Path of the incoming laser pulse, designated by the red arrows. The pulse enters through the port in the top-right of the figure, undergoes compression, and exits through the port in the top-left of the figure. The white lines show the line of sight from the pinhole to the viewport we use for alignment purposes. 
It is important that the focus at the pinhole be as straight and as tight as possible. To achieve this, we adjust Mirror 2 and Mirror 3 such that the laser propagates as close as possible to the horizontal plane containing the pinhole. By also aligning the laser to impinge upon the spherical mirror as normal as possible, the spherical mirror receives and retroreflects the pulse nearly onaxis. As a result, the beam not only focuses straight through the pinhole, but its focal quality improves due to the reduction of spherical aberration.

To verify this, we measured the focal quality at the pinhole by using a moveable CCD camera connected to a Spiricon LBA-PC frame grabber card. We found that the pulses focused by the spherical mirror achieve a focal diameter of about $90 \mu \mathrm{m}$, with no major aberration apparent before or after the focus. The profile of the focus is shown in Fig. 2.6.

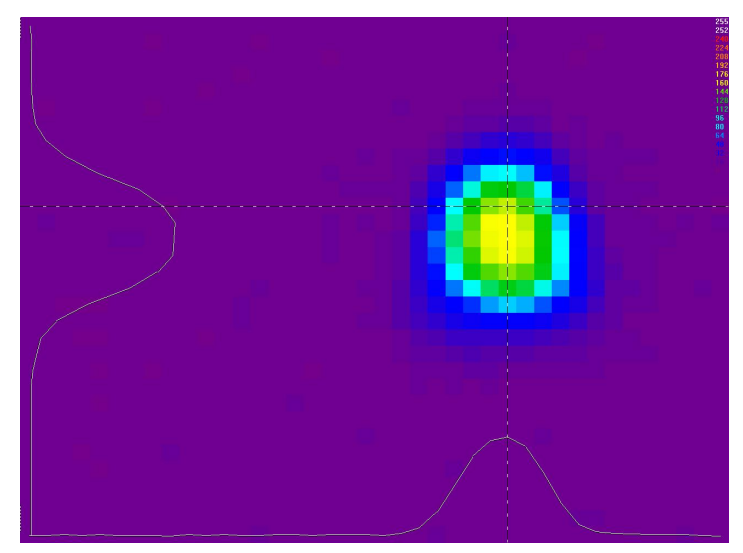

Figure 2.6 CCD image of the laser focus of a one-meter-focus spherical mirror at the pinhole between the two vacuum chambers. The pixel size is $9.8 \mu \mathrm{m}$ by $9.8 \mu \mathrm{m}$.

Unfortunately, the beam alignment changes slightly between each usage of the laser and the vacuum pumps. For this reason, the mount for the spherical mirror features two piezoelectric motors for precision adjustments to the propagation direction of the laser (as seen in the bottomright of Fig. 2.5). These motors are connected via electrical feed-through to a picomotor multi-axis driver (visible in the center of Fig. 2.3), which allows us to direct the beam through the pinhole even when the chambers are sealed. 
To aim the laser, we back-illuminate the pinhole through a window in the interaction chamber. The pinhole is then visible through a viewport into the compression chamber by means of a wellplaced mirror. This way, we can look down the exit port of the compression chamber and visually align the laser spark on the front of the pinhole mount to the pinhole itself using the picomotors. Once the beam is near the pinhole, we maximize the throughput by observing the brightness of the laser flash on a monitor connected to a small camera outside the window of the interaction chamber. (The line of sight to the pinhole through the compression chamber is represented by the white arrows shown previously in Fig. 2.5.)

\subsection{High-Intensity Focus}

Upon entering the interaction chamber through the pinhole, the laser pulse diverges until it reaches an elliptical mirror (produced by Saint-Gobain Crystals). This optic has two foci: the first focus $(8 \mathrm{~cm})$ is positioned below the scattered-photon collection lenses; the second focus $(73 \mathrm{~cm})$ is aligned with the pinhole (hence the need for a tight, straight, aberration-free focus from the compression chamber's spherical mirror). To place the first focus at the center of the interaction chamber, we adjusted the compression of a bellows between the chamber and the pinhole until the distance between the pinhole and the center of the chamber matched the separation of the two mirror foci.

Because of the requirement for relativistic intensities to induce the red-shift in electron radiation, we need to verify that the focused pulse reaches an intensity above $10^{18} \mathrm{~W} / \mathrm{cm}^{2}$. To measure the intensity at the laser focus, we use a time-of-flight spectrometer. This instrument is mounted to the top of the interaction chamber, extending down into the middle of the chamber. This setup is shown in Fig. 2.7.

After pumping down to $10^{-8}$ torr, the interaction chamber is back-filled to $10^{-5}$ torr with argon. 


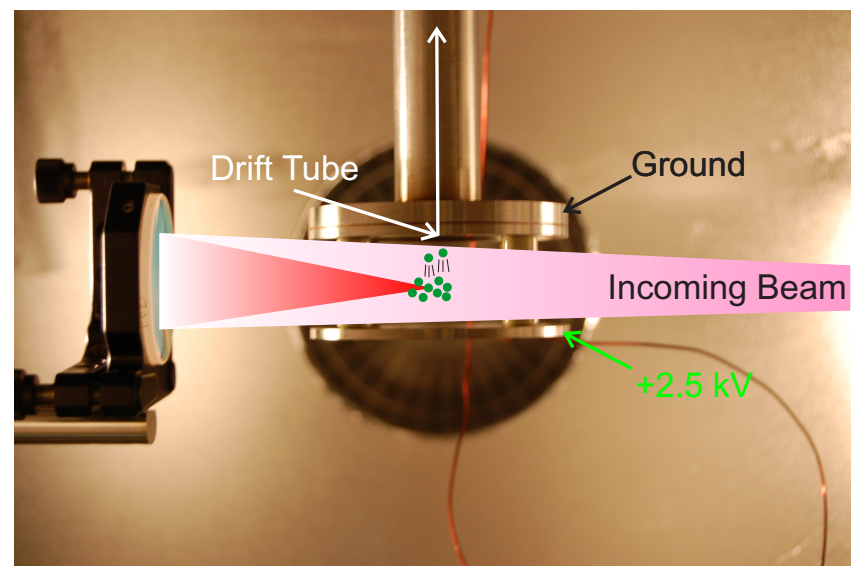

Figure 2.7 Time-of-flight spectrometer extending down into the middle of the interaction chamber in front of the elliptical mirror.

The laser pulse ionizes the argon located at the focus, and the ions reach various positive charge states depending on the intensity experienced at each atom's specific location.

The laser focus is located halfway between two plates held at a potential difference of $2.5 \mathrm{kV}$, as shown in Fig. 2.7. The electric field between the two plates causes the argon ions to accelerate upward until they pass through a copper mesh into a $55 \mathrm{~cm}$-long grounded drift tube. The ions continue upward at constant velocity, and an additional turbo pump helps reduce collisions with ambient molecules. At the end of the tube, a micro-channel plate detector (MCP) is held at $-1.8 \mathrm{kV}$. The ions hit the detector, setting off cascades of electrons detectable externally on an oscilloscope.

Ions with higher charge states accelerate to higher energies in the electric field of the plates. Since the velocities of the ions remain constant once in the drift tube, ions with higher charge states arrive at the MCP sooner than ions with lower charge states. Thus by observing the time delays between the arrival of the laser pulse and the signals from the MCP, we can identify the different charge states of ionized argon achieved in the laser focus. This time delay is given analytically by

$$
t=d \sqrt{m / q V}
$$

where $d$ is the drift tube distance, $m$ is the mass, $q$ is the charge state, and $V$ is the voltage difference between the accelerating plates [17]. 
Each ion charge state can only be induced if the laser reaches some minimum ionization intensity, as shown in Table 2.1. This means that the observation of a given charge state indicates a minimum intensity achieved by the laser. By comparing the delay times predicted by Eq. 2.1 to the delay times displayed on the oscilloscope, we can identify the highest charge state of argon reached in the laser focus. This allows us to infer a lower bound on the peak intensity corresponding to the highest charge state's ionization intensity.

Typically we observe the arrival time $t_{1}$ of the $\mathrm{Ar}^{+}$ions, and then we divide it by $\sqrt{n}$ to predict the arrival time of the $\mathrm{Ar}^{n+}$ ions. These times are compared to the oscilloscope trace in order to identify the highest charge state induced by the laser. The time delays and their corresponding ionization intensities are listed in Table 2.1 [18].

Table 2.1 Time-of-flight time delay and corresponding ionization intensity for argon

\begin{tabular}{ccc}
\hline Charge state of argon & Time delay & Ionization intensity \\
\hline \hline $\mathrm{n}=1$ & $7.1 \mu \mathrm{s}$ & $2.49 \times 10^{14} \mathrm{~W} / \mathrm{cm}^{2}$ \\
\hline $\mathrm{n}=2$ & $5.02 \mu \mathrm{s}$ & $5.80 \times 10^{14} \mathrm{~W} / \mathrm{cm}^{2}$ \\
\hline $\mathrm{n}=3$ & $4.10 \mu \mathrm{s}$ & $1.22 \times 10^{15} \mathrm{~W} / \mathrm{cm}^{2}$ \\
\hline $\mathrm{n}=4$ & $3.55 \mu \mathrm{s}$ & $3.20 \times 10^{15} \mathrm{~W} / \mathrm{cm}^{2}$ \\
\hline $\mathrm{n}=5$ & $3.18 \mu \mathrm{s}$ & $5.06 \times 10^{15} \mathrm{~W} / \mathrm{cm}^{2}$ \\
\hline $\mathrm{n}=6$ & $2.90 \mu \mathrm{s}$ & $7.62 \times 10^{15} \mathrm{~W} / \mathrm{cm}^{2}$ \\
\hline $\mathrm{n}=7$ & $2.68 \mu \mathrm{s}$ & $1.93 \times 10^{16} \mathrm{~W} / \mathrm{cm}^{2}$ \\
\hline $\mathrm{n}=8$ & $2.51 \mu \mathrm{s}$ & $2.61 \times 10^{16} \mathrm{~W} / \mathrm{cm}^{2}$ \\
\hline $\mathrm{n}=9$ & $2.37 \mu \mathrm{s}$ & $1.57 \times 10^{18} \mathrm{~W} / \mathrm{cm}^{2}$ \\
\hline $\mathrm{n}=10$ & $2.25 \mu \mathrm{s}$ & $2.11 \times 10^{18} \mathrm{~W} / \mathrm{cm}^{2}$ \\
\hline
\end{tabular}

With pre-compression pulse energies of $42 \mathrm{~mJ}$, we clearly observed the eighth charge state of argon, corresponding to a laser intensity of at least $2.61 \times 10^{16} \mathrm{~W} / \mathrm{cm}^{2}$. Figure 2.8 displays the 
oscilloscope trace for the various argon ion peaks, each labeled with its corresponding charge state.

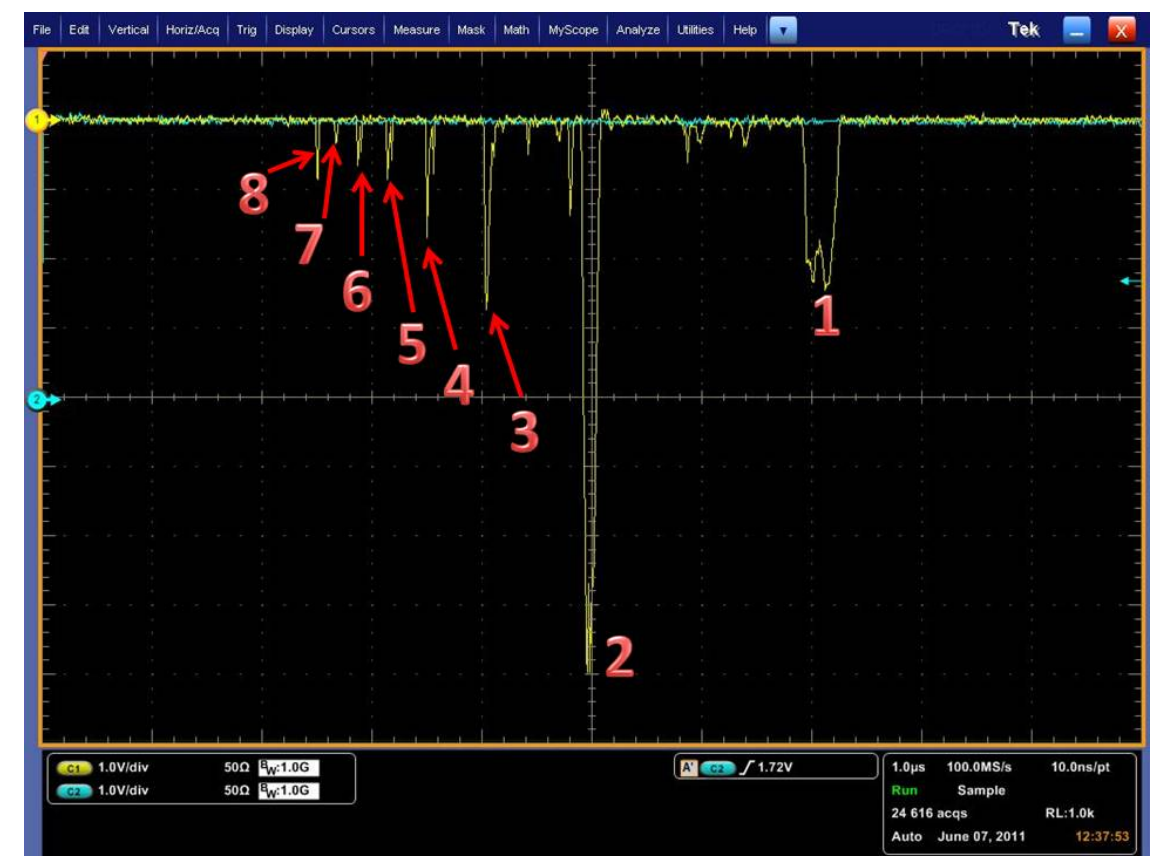

Figure 2.8 Picture of oscilloscope traces showing argon ion peaks $\mathrm{Ar}^{+}$through $\mathrm{Ar}^{8+}$ inside the interaction chamber, $42 \mathrm{~mJ}$ pulse energy.

The ninth and tenth charge states of argon are much harder to see because their ionization volumes are about two orders of magnitude smaller than those of the seventh and eighth charge states. (Treatment of the ionization focal volume as a function of intensity can be found in Appendix B.) Since the signal strength scales with the ionization volume, we use the oscilloscope's histogram mode to look for argon peaks corresponding to the ninth and tenth charge states of argon.

In Fig. 2.9, we note a small peak at $2.37 \mu$ s delay and a prominent peak at $2.25 \mu$ s delay. According to Table 2.1, these correspond to the ninth and tenth charge states of argon, respectively. This estimates the intensity at the focus as $2 \times 10^{18} \mathrm{~W} / \mathrm{cm}^{2}$ - with a pulse energy of only $28 \mathrm{~mJ}$. With room to improve the pulse energy by a factor of two or more, this is strong evidence that we can provide a relativistic environment for accelerating electrons.

Figure 2.10 shows two plots side-by-side for comparison. Figure 2.10(a) shows the histogram 


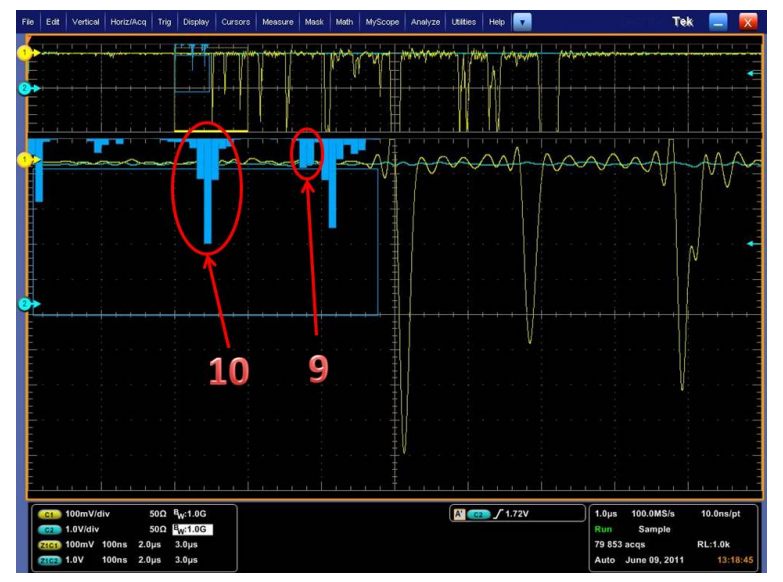

Figure 2.9 Zoomed-in oscilloscope histogram showing peaks from $\mathrm{Ar}^{9+}$ and $\mathrm{Ar}^{10+}$ inside the interaction chamber ( $28 \mathrm{~mJ}$ pulse energy).

data for the laser focusing into the interaction chamber back-filled with argon, while Fig. 2.10(b) shows the histogram data immediately after the argon is shut off and the chamber is evacuated. Note that once the argon is pumped out of the chamber, the peaks corresponding to the ninth and tenth charge states of argon disappear. This provides further evidence that we can indeed reach relativistic intensities in our experiment.

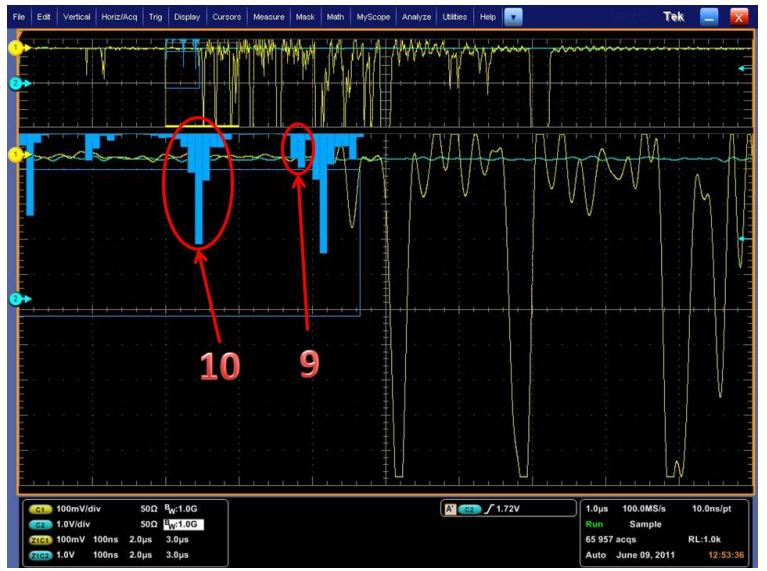

(a) Back-filled argon, 20 microtorr

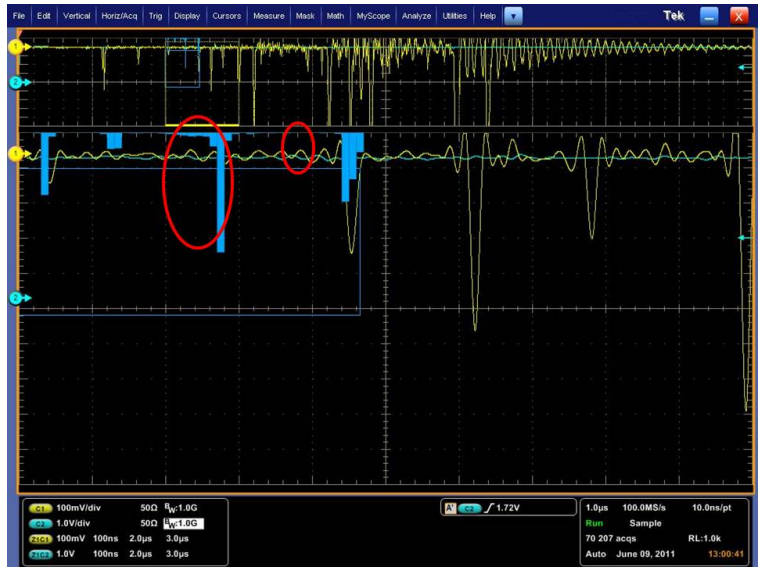

(b) Vacuum background, 470 nanotorr

Figure 2.10 Zoomed-in oscilloscope histograms showing argon ion peaks $\mathrm{Ar}^{9+}$ and $\mathrm{Ar}^{10+}$ compared to vacuum peaks inside the interaction chamber ( $28 \mathrm{~mJ}$ pulse energy). 
Note that these measurements are supported by estimates based on the pulse's energy, duration, and assumed beam waist:

$$
I=\frac{\text { energy }}{\text { area } \cdot \text { time }}=\frac{50 \mathrm{~mJ}}{\pi(5 \mu \mathrm{m})^{2} \cdot(35 \mathrm{fs})}=2 \times 10^{18} \mathrm{~W} / \mathrm{cm}^{2}
$$

\subsection{Vacuum and Gas Systems}

We require low pressures in the interaction chamber to limit radiation from contaminating molecules in the chamber. We achieve these pressures through differential pumping.

Both chambers are fitted with a turbo pump: the Turbo-V 301 Navigator attached to the compression chamber is capable of pumping speeds up to $250 \mathrm{l} / \mathrm{s}$, and the Turbo-V 551 Navigator attached to the interaction chamber is capable of pumping speeds up to $600 \mathrm{l} / \mathrm{s}$. Because the pinhole largely frustrates diffusion between the two chambers, we can use the turbo pump of the compression chamber to back the turbo pump of the interaction chamber. In this configuration, the scroll pump backs only the compression chamber's turbo pump, and we are able to reach pressures of $10^{-8}$ torr in the interaction chamber. This is measured using an inverted magnetron gauge on the opposite side of the interaction chamber from the turbo pump and the gas intake valve.

Other common practices are employed to create and maintain high vacuum. We take standard precautions to preserve a clean environment inside the chambers. We cut notches into the posts and bolts to release air trapped in bolt threads and other pockets. We use copper gaskets and nickel washers to make tight, metal-on-metal seals between components. We bake the interaction chamber using two heating straps capable of reaching $200^{\circ} \mathrm{C}$ in order to expedite the outgassing process. We also use a gate valve between the compression chamber's turbo pump and the scroll pump to correct a problem of particulate contamination through improper venting.

Because the pinhole allows a pressure differential between the two chambers, precautions must be taken to prevent the bellows from shortening and moving the chambers. We immobilized the 
compression chamber and the interaction chamber by bolting down aluminum clamps machined to fit around the feet of each chamber. In addition, we installed an appropriately-sized metal rod between the two flanges of the bellows to maintain the desired separation.

We use helium as the donor of the electrons needed for our experiment. Since helium loses its second electron already at $8.7 \times 10^{15} \mathrm{~W} / \mathrm{cm}^{2}$, ionization in the laser focus occurs well in advance of the maximum intensity of the pulse. These electrons thus become the relativistically-accelerated 'free electrons' which we wish to study in our experiment.

To have two helium atoms present on average in the highest intensity region of the laser focus, we back-fill the chamber to $10^{-6}$ torr. This is done by introducing the gas through a leak valve into the port opposite from the inverted magnetron pressure gauge. This way, we can gradually introduce gas into the chamber while accurately reading the pressure inside the chamber.

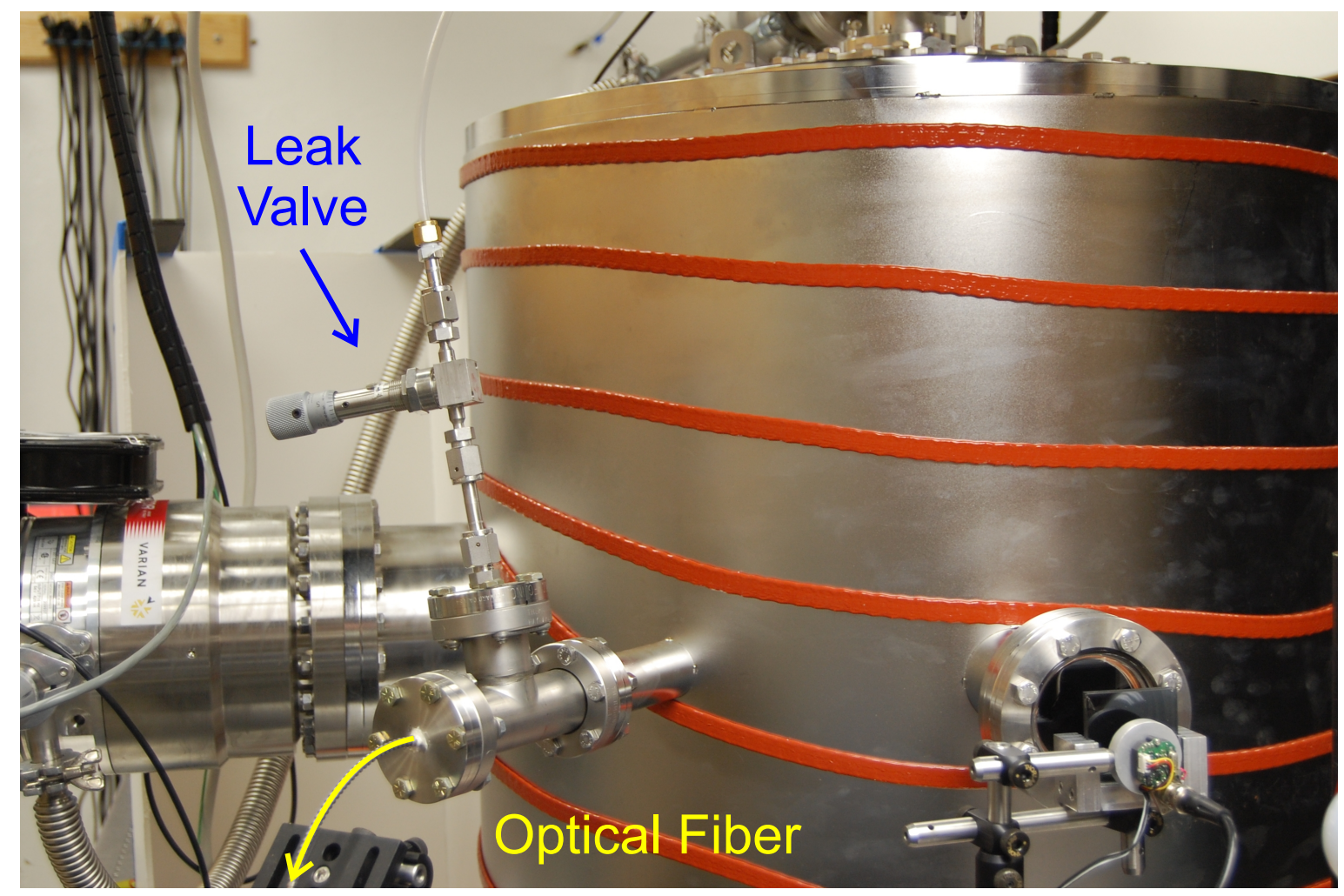

Figure 2.11 The incoming gas line connected to a leak valve. This valve shares a port of the interaction chamber with the out-coming optical fiber. 


\subsection{Collection of Electron Radiation}

Once the interaction chamber is back-filled with helium, the laser liberates and accelerates the electrons, causing them to radiate. We collect the electron photoemission out the lateral side of the laser focus using one-to-one imaging, as shown in Fig. 2.12(a). The lenses image the laser focus to the end of an optical fiber. The associated lens tubes are attached to a cage system connected to an external $\mathrm{XYZ}$ positioner, which is mounted atop the interaction chamber. This allows us to adjust the point of collection to coincide with the laser focus. By referencing the plasma streak at the focus in the unevacuated chamber (seen in Fig. 2.12(b)), we can optimize the positioning of the collection lenses.

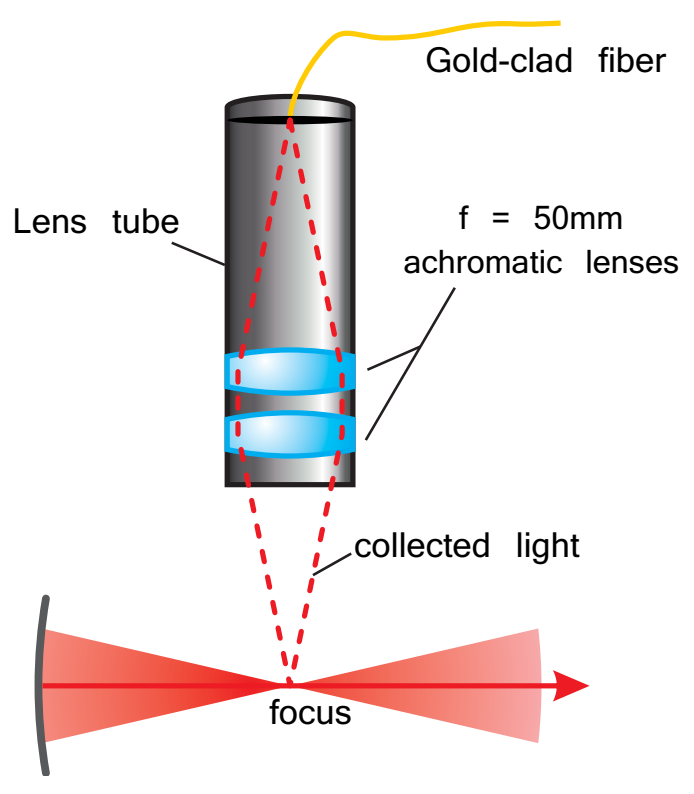

(a) Schematic for one-to-one imaging

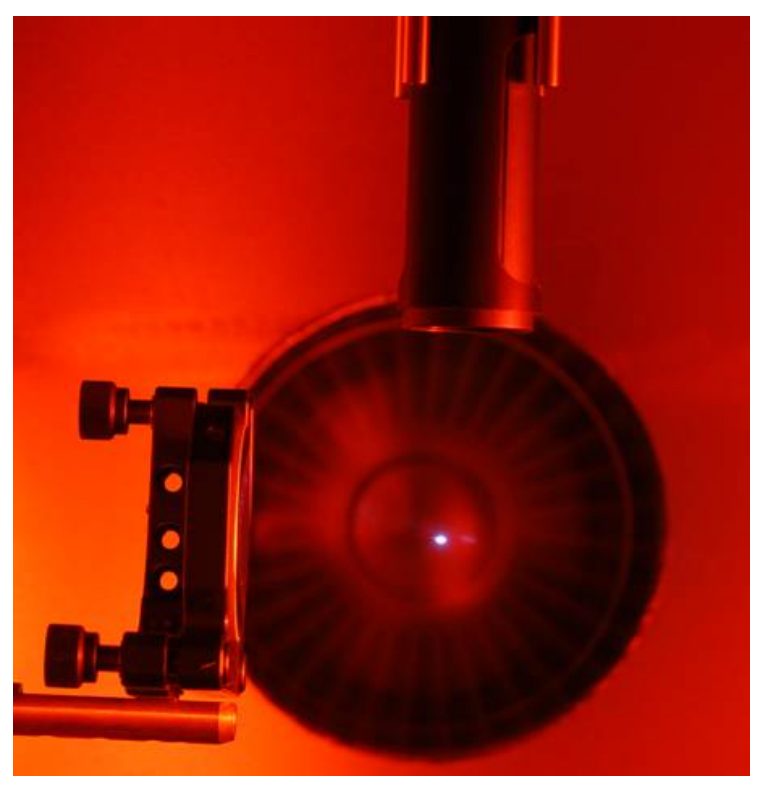

(b) Ionization at the laser focus

Figure 2.12 Collection of photoemission from lateral side of the laser focus using two lenses attached to an external XYZ positioner. 


\subsection{Temporal Filtering}

The collection lenses gather both electron photoemission and light scattered from the walls of the chamber. This is the reason for the large size of the interaction chamber $(76 \mathrm{~cm}$ tall, $71 \mathrm{~cm}$ in diameter): the laser pulse must traverse a significantly-farther distance in traveling to and returning from the chamber wall before encountering the collection lenses. As a result, the electron photoemission precedes the earliest laser reflections by over $2 \mathrm{~ns}$ - a time easily distinguishable by the time digitizer. Once the arrival time of the laser pulse is identified, any signal outside this time range can be ignored as a reflection.

To determine the arrival time of the laser pulse, we placed a piece of glass immediately below the collection system, reflecting the laser directly through the lenses into the fiber. We inserted numerous neutral-density filters to protect the avalanche photodiode, attenuating the signal enough so that only the original pulse profile was visible. This pinpointed the arrival time of the pulse. Figure 2.13 shows that the laser pulse arrives about 139 ns after the external trigger.

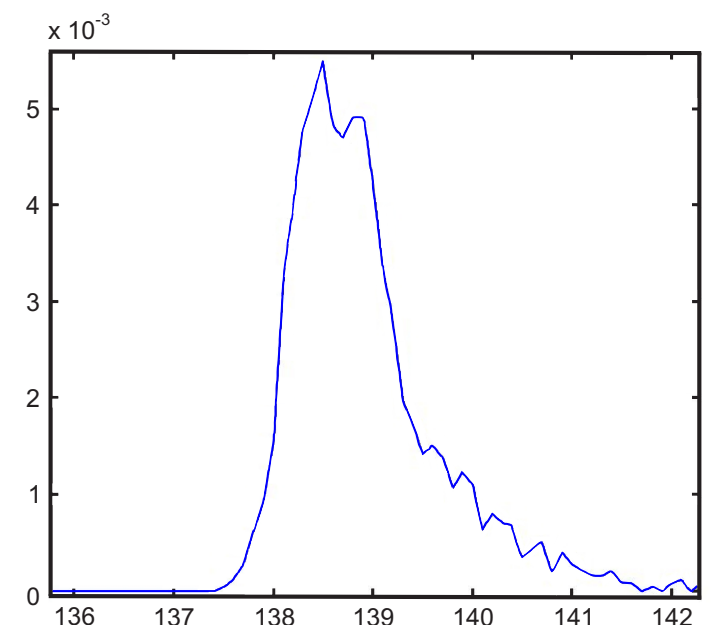

Figure 2.13 Direct reflection of laser pulse into collection device, used to determine time window corresponding to the arrival of the laser pulse (in ns).

In Section 3.1, we use this information to identify the arrival of the laser pulse in a measurement of the noise background. 


\subsection{Efficient, Light-Tight Optical Fiber}

We need a protected detection channel to minimize losses to the photoemission signal and prevent noise from entering the system. We use a custom multi-mode fiber with a core diameter of $105 \mu \mathrm{m}$ and an exterior gold buffer of $30 \mu \mathrm{m}$. This barrier provides protection against light leakage through the walls of the fiber. (The imaginary part of the index of refraction $\kappa$ for gold at $800 \mathrm{~nm}$ is 4.2 [19]; the skin depth is $\delta=\lambda / 2 \pi \kappa$ [20], or, in this case, a mere $30.3 \mathrm{~nm}$.) With a thickness of nearly 1,000 skin depths, the gold buffer completely prevents laser light from leaking into the fiber.

Each end of the optical fiber is terminated with a standard FC connector. Both fiber ends were polished manually using aluminum oxide polishing sheets, with grit sizes ranging from $10 \mu \mathrm{m}$ to $0.3 \mu \mathrm{m}$. Inspection of each termination with a 200-times magnifier revealed surface smoothness that permits high signal transmission.

Beginning from the one-to-one imaging setup, the fiber passes from the chamber's center into a port in the interaction chamber wall. From there, the fiber exits the chamber through a drilledout flange hole patched with Torr Seal. A protective metal jacket supports the fiber outside the chamber until terminating at a fiber launch (shown right-of-center in Fig. 2.14). At this point, the fiber's enclosed signal is ready for spectral filtering.

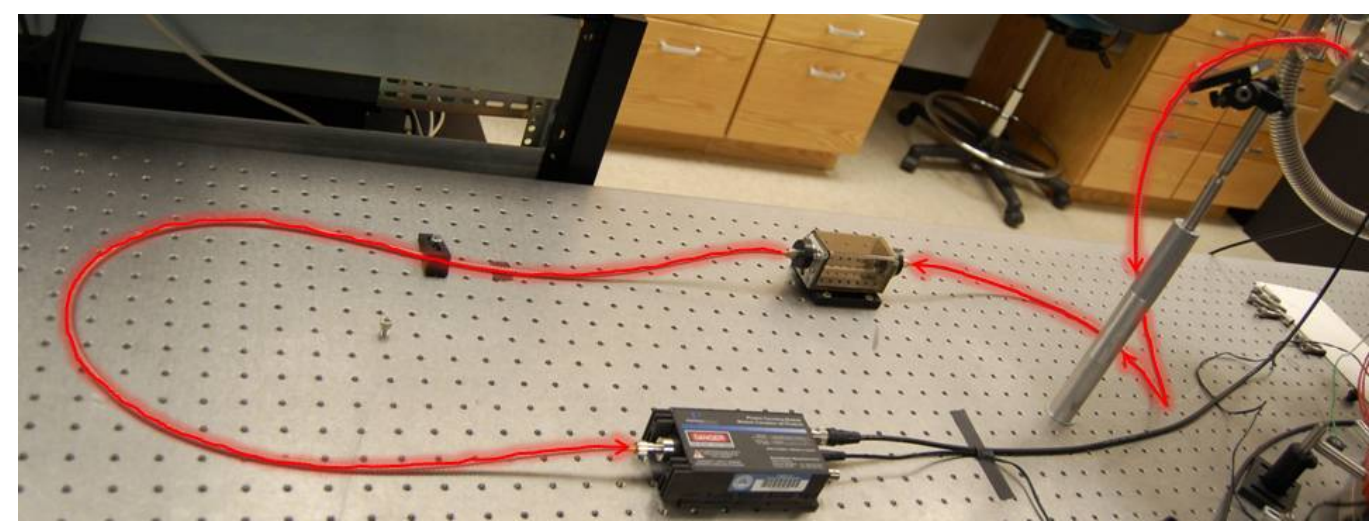

Figure 2.14 The optical fiber exiting from the chamber feed-through (top-right corner) and into the spectral filter bench. From there, the light is sent to the avalanche photodiode for detection (middle-bottom). 


\subsection{Spectral Filtering}

The optical fiber from the light collection system carries a mixed signal comprised of the redshifted photons from electron photoemission and the fundamental-wavelength photons from the laser. Because the avalanche photodiode cannot distinguish between photons of different wavelengths, we must take steps to eliminate laser photons from the signal before passing it to the detector. We accomplish this using spectral filtering.

Three spectral filters are used to discriminate against photons from the laser: one bandpass filter and two identical high-pass filters. The manufacturer's transmission specifications for both types of filters are shown as a function of wavelength in Fig. 2.15. Each filter reduces the amount of $800 \mathrm{~nm}$ light by orders of magnitude while simultaneously transmitting $70 \%$ to $80 \%$ of the red-shifted $900 \mathrm{~nm}$ light.

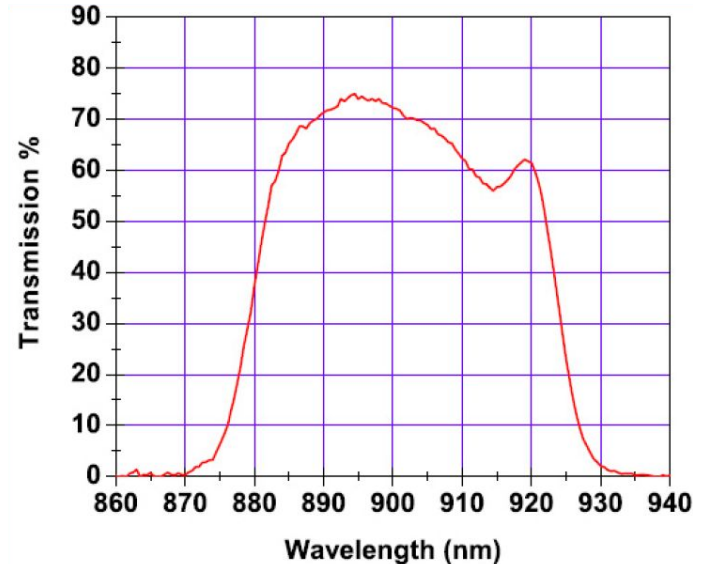

(a) Bandpass filter transmission specification

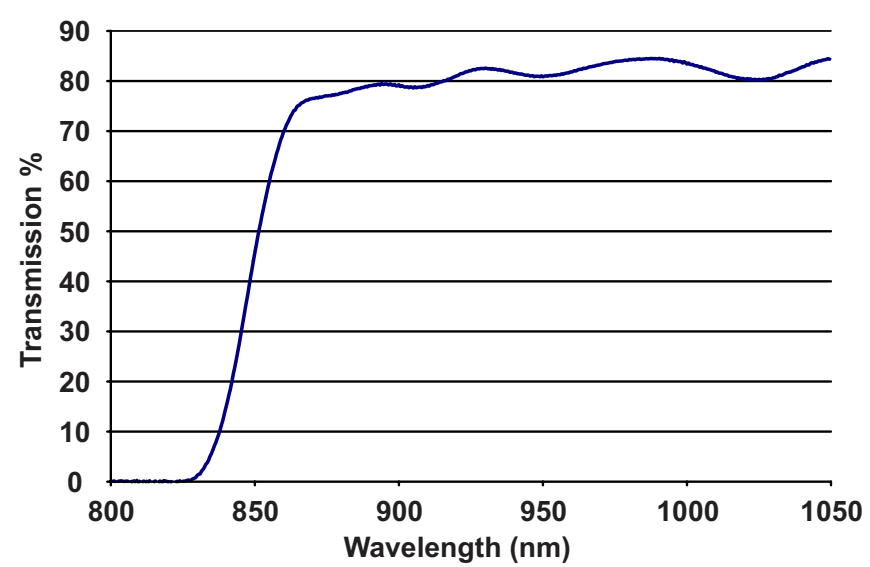

(b) High-pass filter transmission specification

Figure 2.15 Manufacturer's transmission specifications of the spectral filters as a function of wavelength.

In order to use the spectral filters properly, we collimate the light using a Thorlabs FiberPort, which allows precise control over six degrees of freedom. The filters are inserted here before 
the light is focused back into another optical fiber using a second FiberPort. This setup is shown in Fig. 2.16. During typical use, the fiber bench is enclosed by a light-tight cover to prevent contamination by ambient photons.

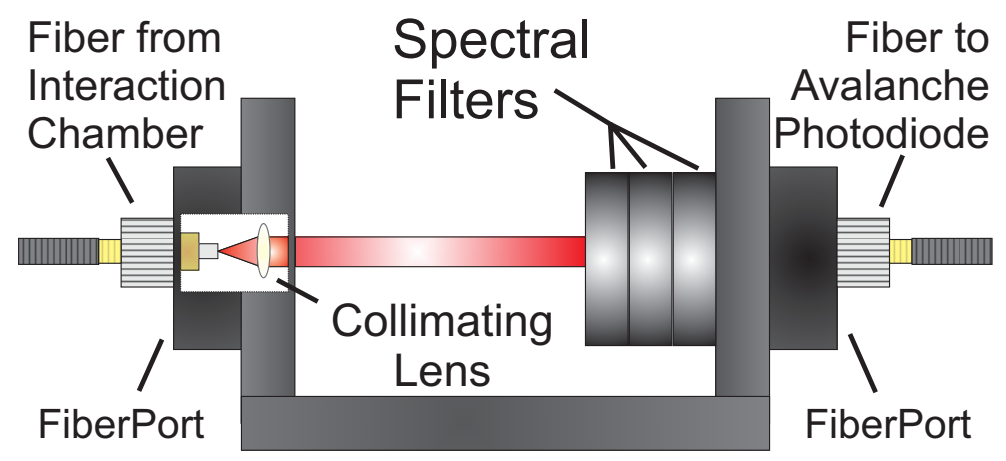

Figure 2.16 Schematic for the spectral filter box.

We align the FiberPorts such that the light exits the fiber, collimates, and relaunches into a second fiber with $95 \%$ efficiency in the red-shifted wavelength range. This was measured by shining light from a $900 \mathrm{~nm}$ diode laser into the collection device inside the interaction chamber. This provided us with $3.9 \mathrm{~mW}$ of $900 \mathrm{~nm}$ light entering the filter system. We adjusted the coupling device until we could detect $3.7 \mathrm{~mW}$ of the $900 \mathrm{~nm}$ light coming out of the fiber on the receiving side of the filter system.

In Section 3.1, we show the effect of these spectral filters on the noise background.

\subsection{Detection System}

Once the signal is filtered and coupled back into the second optical fiber, it is ready for detection. We use a PerkinElmer SPCM-AQR-14-FC avalanche photodiode because it is capable of registering effects from single photons. The manufacturer's specification of the photon detection efficiency of this instrument is shown in Fig. 2.17. This suggests that we can detect red-shifted photons with a $35 \%$ efficiency. 


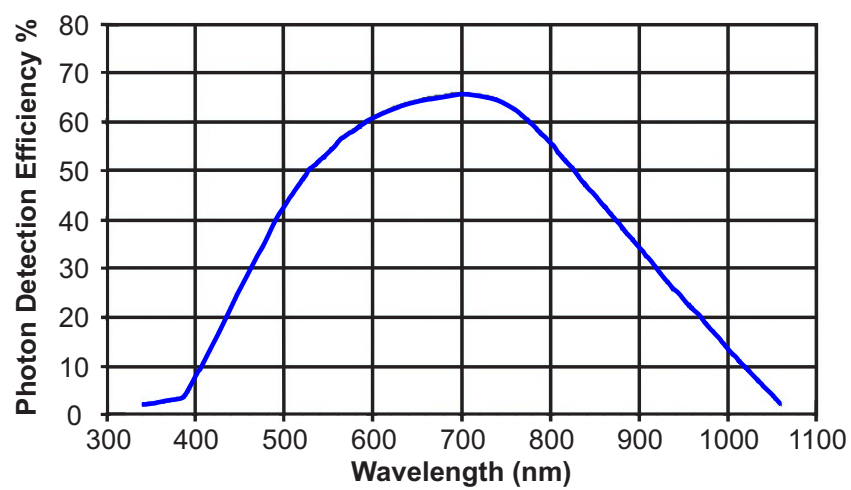

Figure 2.17 Manufacturer's specification of photon detection efficiency of the avalanche photodiode as a function of wavelength.

When a photon is detected, the avalanche photodiode sends a TTL signal to an Ortec 9353 Time Digitizer, which is capable of 100-ps time resolution. The arrival of these photons is time-stamped relative to the trigger received directly from the pulse generator of the laser. The computer counts and displays the number of photons detected during each $0.1 \mathrm{~ns}$ time bin. 


\section{Chapter 3}

\section{Experimental Results}

In this chapter, we report on the effect of spectral filtering on the noise background, including the number of photons counted during the timing window corresponding to the laser pulse. We also outline goals for future investigation.

\subsection{Effect of Spectral Filtering}

To determine the effect of spectral filtering, we first need to measure the unfiltered noise background collected into the optical fiber. This is done by collecting photons scattered from the laser focus inside the evacuated interaction chamber directly below the collection lenses. The collected photons can trigger the avalanche photodiode, which sends a signal recorded by the time digitizer. These individual counts accumulate in the 0.1 ns-time bins over many laser shots, and they outline the time profile of the noise background.

Unfortunately, we cannot observe the entire unfiltered background at once because an avalanche photodiode has a $50 \mathrm{~ns}$ 'dead time' after the detection of a photon. This means that we can only see one photon per shot of the laser before the detector becomes blind to any ensuing photons. With no filters present, this will always happen many nanoseconds before the arrival of the pulse, 
effectively preventing any measurement of the noise background during the direct signal window. Thus to measure the full noise profile, we must reconstruct it by sampling the background in multiple segments. We accomplish this by using successively-greater orders of neutral-density filters in taking photon measurements.

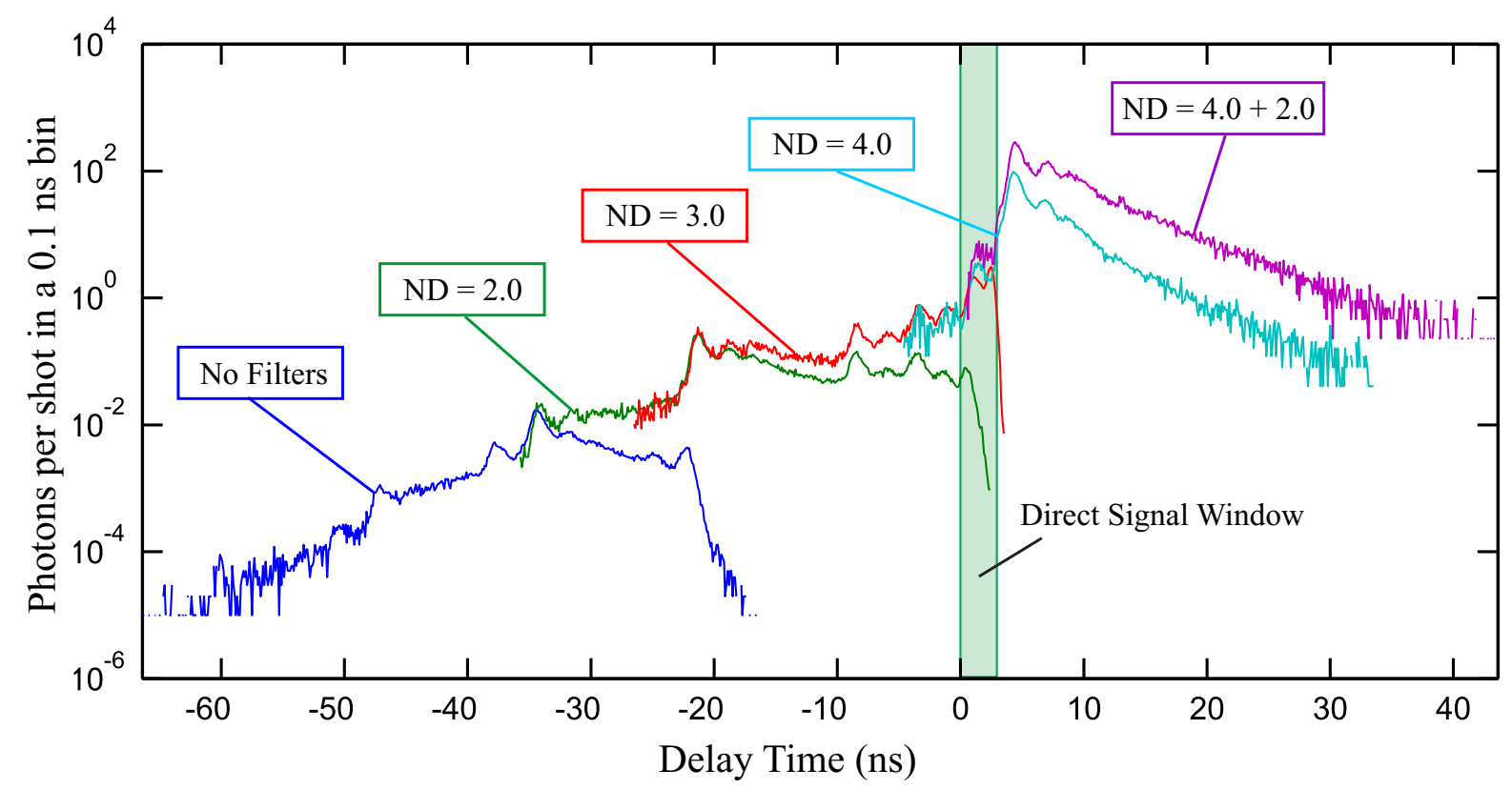

Figure 3.1 Example of reconstruction of noise background using neutral-density filters. Time delay zero signifies the arrival of the laser pulse.

Figure 3.1 shows an example of this reconstruction process. The first segment of the background signal (blue) is measured using no filters. After aggregating many photon counts, we have good statistics for this portion of the background. Notice that at a delay time of about $-33 \mathrm{~ns}$, the histogram shows a steady decline in photon counts, meaning that the avalanche photodiode is dead too often to continue detecting the true magnitude of the noise background. This is where we must start measuring the second segment of the full noise profile.

To determine the strength of filters needed to begin this next segment, we find the difference between the previous segment's lowest and highest photon count rates. (In the case of Fig. 3.1, this is about three orders of magnitude.) We then insert various neutral-density filters that collectively 
match the order of magnitude of this growth. The filters eliminate the lower-level noise detected during the first segment, thus allowing the avalanche photodiode to begin recording photons at the higher amplitudes where the previous segment became unreliable. (In practice, we typically insert too little attenuation so the segments overlap each other, thus ensuring that we reconstruct the background accurately. Each additional segment of Fig. 3.1 demonstrates this technique.) We repeat this process several times until we measure the noise coincident with the arrival of the laser pulse and its reflections.

Because each segment is recorded using a different amount of attenuation, we must scale the photon measurements by dividing each one by the strength of its associated filters. We also divide by the assumed collection efficiency (80\%) and detection efficiency (30\%) to complete our estimation. After normalizing the measurements to display the number of detected photons per shot, we stitch the overlapping segments together to create a panoramic image of the unfiltered noise background. The complete reconstruction of the noise background with the spherical silver mirror is shown by the upper (red) curve in Fig. 3.2.

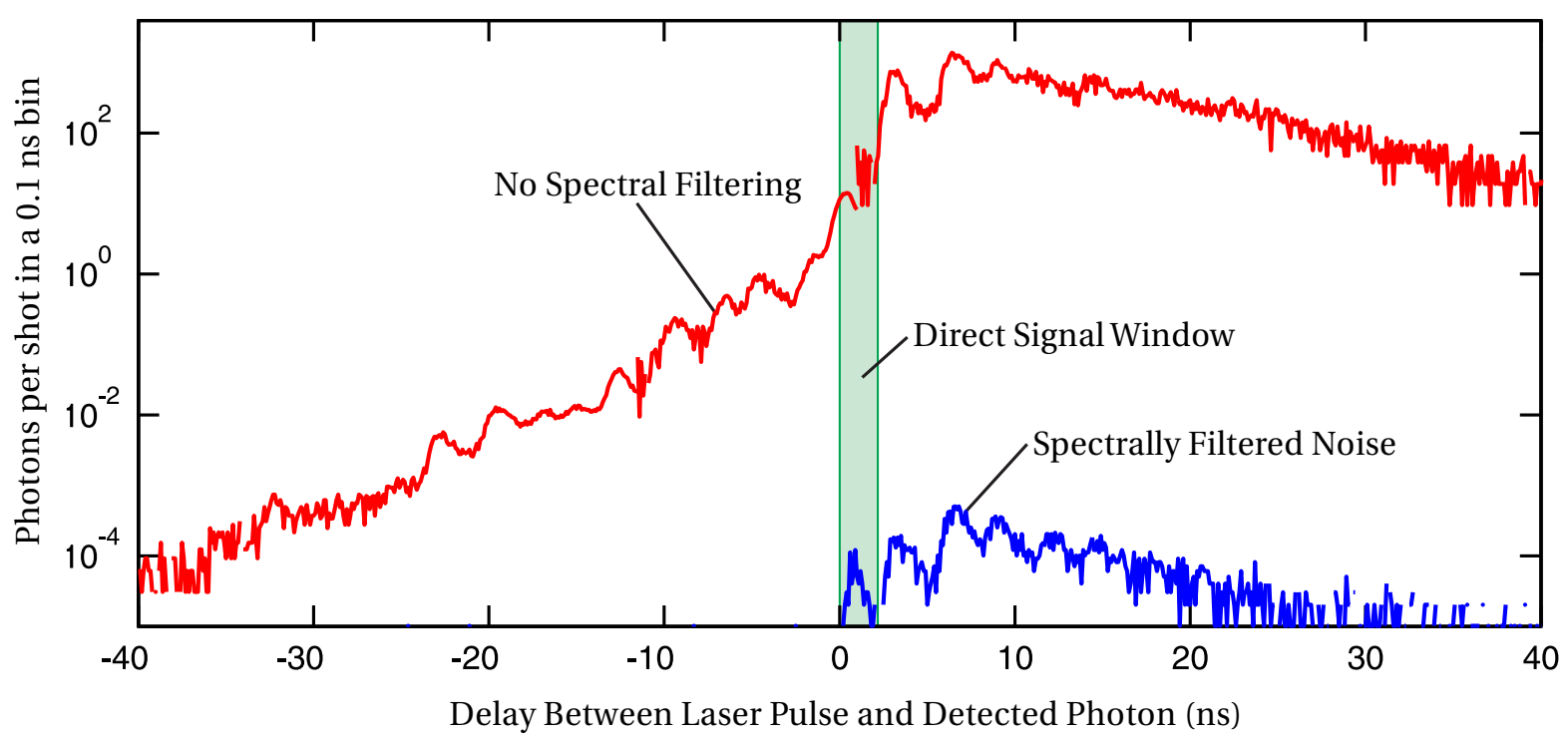

Figure 3.2 Reduction of noise background due to spectral filtering. The arrival of the laser pulse is denoted by the vertical lines. 
Only needing to replace the neutral-density filters with the bandpass filter and both high-pass filters, we use the same general setup to measure the effectiveness of the spectral filters at reducing noise background. After hours of accumulation, we scale the amplitude of the resulting histogram using the same assumptions as before for the collection and detection efficiencies. This measurement - also taken with the spherical silver mirror — is represented by the lower (blue) curve in Fig. 3.2. (Note that time delay zero marks the measured arrival of the laser pulse, according to the data displayed in Fig. 2.13 of Section 2.7.)

These results suggest that the spectral filters reduce the noise background by nearly six orders of magnitude during the timing window. In addition to the spectral filtering, the temporal filtering reduces the noise floor even further because we can completely disregard any photons detected after the direct signal window. Note also that essentially no photons were detected during the $50 \mathrm{~ns}$ before the timing window (only 4 clicks in 324,003 shots). This means that the avalanche photodiode is almost never blind during the direct signal window.

We repeated this noise background measurement after replacing the spherical silver mirror with the elliptical dielectric mirror. Because the dielectric mirror scatters less light than the silver mirror, we detect even fewer photons than before in the direct signal window while spectrally filtering the signal. Figure 3.3 displays the unfiltered noise background from the elliptical mirror and its attenuation by the three spectral filters. (For this measurement, we did not re-measure the arrival time of the laser pulse. This means that the interval displayed in Fig. 3.3 is only the anticipated direct signal window, estimated by measuring the spacing between features common to the noise profiles in Fig. 3.2 and Fig. 3.3.) 


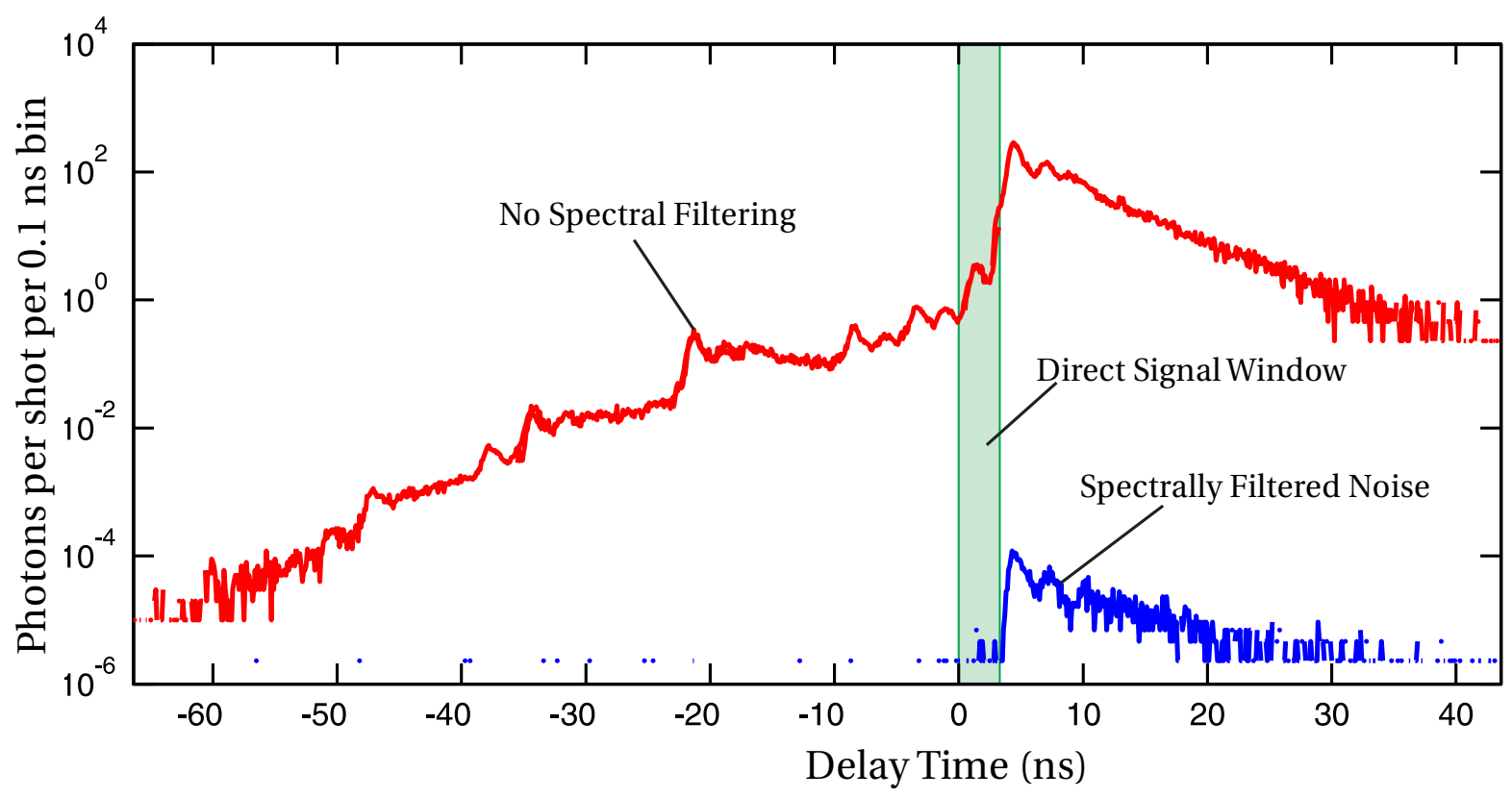

Figure 3.3 Reduction of noise background (elliptical mirror) due to spectral filtering. The vertical lines only suggest the anticipated time of pulse arrival.

\subsection{Comparison to Predicted Red-Shifted Photon Count}

It is crucial that we compare the noise background photon count in Section 3.1 to the predicted red-shifted photon count in Section 1.3.1. If we integrate the filtered noise background data over the timing window of the pulse's arrival in Fig. 3.3, we find that 24 photons were detected in this time interval over the course of 432,003 shots.

Theory predicts that 1 red-shifted photon will be emitted every 300 shots into the solid angle of the collection lenses inside the interaction chamber [4]. To calculate the number of red-shifted photons we expect to detect after the same number of shots, we must make some assumptions about the efficiency of the experimental apparatus. First, we assume that light radiated into the solid angle of the one-to-one imager is collected into the fiber with $80 \%$ efficiency. Next, we assume that the spectral filters transmit the $900 \mathrm{~nm}$ light with 65\% efficiency, and that the FiberPort couples back

into the second fiber with 95\% efficiency. Finally, we assume that the avalanche photodiode detects 
$900 \mathrm{~nm}$ light with $30 \%$ efficiency. Using these numbers, we can calculate the expected number of red-shifted photons counted after 432,003 laser shots:

$$
(432,003 \text { shots })\left(\frac{1 \text { photon }}{300 \text { shots }}\right)(.8)(.65)(.95)(.3)=213 \text { photons }
$$

In terms of photon count rates, this means that we expect to count a red-shifted photon every 2,028 shots, while we expect to detect a noise photon every 18,000 shots. Thus even with these conservative efficiency estimates, we predict that the photoemission signal from the accelerated electrons in the lateral direction is nearly ten times as strong as the detected noise floor. This should be enough for us to distinguish the electron radiation from the noise background.

\subsection{Conclusions}

In summary, we were able to achieve intensities of $2 \times 10^{18} \mathrm{~W} / \mathrm{cm}^{2}$, which is strong enough to accelerate the electrons to mildly-relativistic speeds. Using spectral filters, we reduced the noise background during the direct signal window by over six orders of magnitude. As a result, the predicted red-shifted photon count rate in the lateral direction is bigger than this filtered noise background by nearly an order of magnitude.

\subsection{Future Work}

\subsubsection{Preparation for Electron Radiation Experiment}

While these results are informative, more work and investigation remains before proceeding with the performance of the electron radiation experiment. As part of the preparation process, we must probe the noise background further to ensure that we can properly distinguish between noise and red-shifted photoemission. We first performed the intensity and noise measurements of our system 
with the less-expensive spherical silver mirror in the experimental chamber. Now that we better appreciate the feasibility of our measurement, we have installed the elliptical mirror and have begun re-evaluating the intensity and noise measurements, but further investigation is necessary.

Once we are convinced the system is prepared to perform the electron radiation experiment, we will turn up our laser to run at pulse energies as high as $100 \mathrm{~mJ}$. This high performance level is unsustainable because the increased energies can damage optics in the laser system. Therefore, this is the last step before we focus the laser into the helium and look for red-shifted photons.

\subsubsection{Parametric Down-Conversion}

Another plan for the future is to use parametric down-conversion to measure the absolute efficiency of the collection, filtering, and detection apparatus. After using a crystal to down-convert one photon into two entangled photons, one photon of the pair is immediately detected. The other photon, whose wavelength is $900 \mathrm{~nm}$, passes through the collection and filtering systems before detection. Since the two photons are entangled, the detection of the first photon means that a second photon entered the collection system. Thus we can estimate the overall efficiency of the system by comparing the detected number of $900 \mathrm{~nm}$ photons to the number of immediately-counted photons. A schematic for this setup is shown in Fig. 3.4.

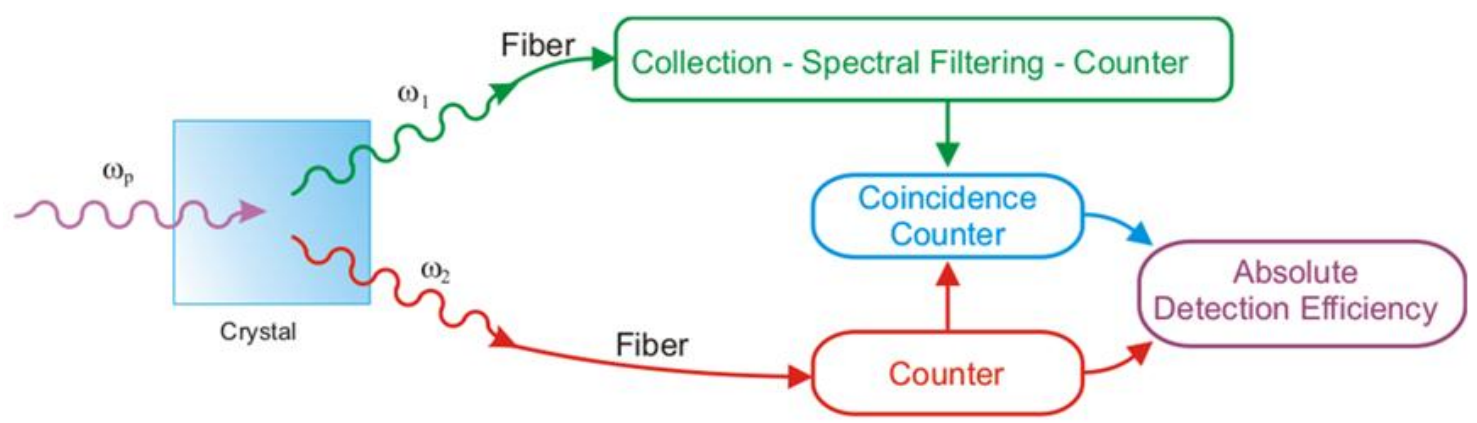

Figure 3.4 Schematic for down-conversion setup used to measure absolute efficiency of collection and filtering system. 
The equipment necessary for this measurement is already at hand. We will use a crystal that converts photons from an argon laser into two photons, one of which has a wavelength of $900 \mathrm{~nm}$. Up to this time, much effort has gone into putting the argon laser system into working order by wiring it for power, hooking it up to a chilled water system, and fixing its pumping system. We have not yet assembled the down-conversion setup.

\subsubsection{Spectral Make-up of Background}

Another possible assessment of the experimental apparatus involves obtaining a monochromator to determine the spectral make-up of the noise background passing through the spectral filters. Because of the single-photon nature of this noise (only 1 photon every 18,000 shots, according to data shown in Fig. 3.3), the monochromator must have very high throughput for the measurement to be feasible. The filtered noise background could result from a trace amount of $800 \mathrm{~nm}$ light surviving through all three spectral filters, or it may be other wavelengths of light from vacuum contamination, atomic radiation, the laser, or some other unidentified source. By using a monochromator to scan the background for its spectral make-up, we could potentially identify any sources of the existing noise. This could lead to preventative measures limiting the noise background even further.

\subsubsection{Signal Strength and Interpretation Issues}

It is possible that the ponderomotive gradient of the laser focus is so strong that electrons are forced from the focus too quickly to radiate to the extent predicted by Peatross et al. in 2008 [4]. If this

prevents us from seeing a signal at $10^{-6}$ torr of helium, we may resort to increasing the gas density in order to increase the signal strength. Since we are attempting to measure radiation from single electron wave packets, this could foreseeably hinder our efforts in interpreting the signal. In order to improve our ability to create and detect a strong signal, we may in the future consider: using a mirror with a shorter focus to achieve higher intensities; fine-tuning the XYZ positioner to hone 
in on the laser focus; adding magnets near the laser focus to prevent electrons from hitting the collection lenses; or re-polishing the optical fiber termination in the interaction chamber to remove any possible degradation.

Since both electrons from a helium atom emerge at similar intensities, it is also possible that we will not be able to see single electron photoemission at all. Instead, we may only observe radiation from two electrons traveling on trajectories that are similar, but separate. In the future, we may look into different solutions for this potential problem. 


\section{Appendix A}

\section{Scattering by a Current Distribution}

To study the radiation from an extended charge distribution in an external field, we model a Gaussian current distribution driven by a plane wave (polarized in the $\hat{z}$-dimension and propagating in the $x$-direction) in the low-intensity limit [4]. This is given by

$$
\vec{J} \propto \hat{z} r_{0}^{-3} e^{-r^{2} / r_{0}^{2}} e^{i(k x-\omega t)}
$$

where $k$ is the wave number, $\omega$ is the frequency of the plane wave, $t$ is time, and $r_{0}$ relates to the current distribution size.

In the case of a current density $\vec{J}$ without a charge density $\rho$, Jefimenko's generalization of Coulomb's law is given [21] by

$$
\vec{E}(\vec{r}, t)=\frac{1}{4 \pi \varepsilon_{0}} \int d^{3} r^{\prime}\left\{-\frac{1}{c^{2}\left|\vec{r}-\vec{r}^{\prime}\right|}\left[\frac{\partial \vec{J}\left(\vec{r}^{\prime}, t^{\prime}\right)}{\partial t}\right]_{\mathrm{ret}}\right\}
$$

where $\left|\vec{r}-\vec{r}^{\prime}\right|$ is the distance from the source to the field. Inserting Eq. A.1 into Eq. A.2 yields

$$
\vec{E}(\vec{r}, t) \propto \int d^{3} r^{\prime}\left\{-\frac{1}{c^{2}\left|\vec{r}-\vec{r}^{\prime}\right|}\left[\hat{z}(-i \omega) r_{0}^{-3} e^{-r^{\prime 2} / r_{0}^{2}} e^{i\left(k x^{\prime}-\omega t^{\prime}\right)}\right]_{\mathrm{ret}}\right\}
$$

Next, evaluate at the retarded time:

$$
\vec{E}(\vec{r}, t) \propto \int d^{3} r^{\prime}\left\{-\frac{1}{c^{2}\left|\vec{r}-\vec{r}^{\prime}\right|}\left[\hat{z}(-i \omega) r_{0}^{-3} e^{-r^{\prime 2} / r_{0}^{2}} e^{i\left(k x^{\prime}-\omega\left[t-\left|\vec{r}-\vec{r}^{\prime}\right| / c\right]\right)}\right]\right\}
$$


Since we are interested only in the electric field far removed from the source, we expand $\left|\vec{r}-\vec{r}^{\prime}\right|$ in the limit that $r \gg r^{\prime}$ :

$$
\begin{aligned}
\left|\vec{r}-\vec{r}^{\prime}\right| & =\sqrt{\left(x-x^{\prime}\right)^{2}+\left(y-y^{\prime}\right)^{2}+\left(z-z^{\prime}\right)^{2}}=\sqrt{x^{2}+y^{2}+z^{2}-2\left(x x^{\prime}+y y^{\prime}+z z^{\prime}\right)+\ldots} \\
& =r \sqrt{1-\frac{2\left(x x^{\prime}+y y^{\prime}+z z^{\prime}\right)}{r^{2}}+\ldots} \approx r-\frac{\left(x x^{\prime}+y y^{\prime}+z z^{\prime}\right)}{r}
\end{aligned}
$$

We use both terms of the approximation in the exponent, and we keep only the first term in the denominator. Remembering the dispersion relation $\omega=c k$, we rearrange factors to see that

$$
\begin{aligned}
\vec{E}(\vec{r}, t) \propto \hat{z} \frac{(i \omega) r_{0}^{-3}}{c^{2} r} e^{i(k r-\omega t)} \int d^{3} r^{\prime}\left\{\exp \left[-\frac{r^{\prime 2}}{r_{0}^{2}}+i k\left(x^{\prime}-\frac{\left(x x^{\prime}+y y^{\prime}+z z^{\prime}\right)}{r}\right)\right]\right\} \\
=\hat{z} \frac{(i \omega) r_{0}^{-3}}{c^{2} r} e^{i(k r-\omega t)} \int_{-\infty}^{+\infty} d x^{\prime}\left\{\exp \left[-\frac{x^{\prime 2}}{r_{0}^{2}}+i k\left(1-\frac{x}{r}\right) x^{\prime}\right]\right\} \\
\int_{-\infty}^{+\infty} d y^{\prime}\left\{\exp \left[-\frac{y^{\prime 2}}{r_{0}^{2}}-i k \frac{y}{r} y^{\prime}\right]\right\} \\
\int_{-\infty}^{+\infty} d z^{\prime}\left\{\exp \left[-\frac{z^{\prime 2}}{r_{0}^{2}}-i k \frac{z}{r} z^{\prime}\right]\right\}
\end{aligned}
$$

Complete the square in the exponents in each integrand:

$$
\begin{aligned}
\vec{E} \propto \hat{z} \frac{(i \omega) r_{0}^{-3}}{c^{2} r} e^{i(k r-\omega t)} & \int_{-\infty}^{+\infty} d x^{\prime}\left\{\exp \left[-\left(\frac{x^{\prime}}{r_{0}}-i \frac{k r_{0}}{2}\left(1-\frac{x}{r}\right)\right)^{2}\right]\right\} \exp \left[-\frac{k^{2} r_{0}^{2}}{4}\left(1-\frac{x}{r}\right)^{2}\right] \\
& \int_{-\infty}^{+\infty} d y^{\prime}\left\{\exp \left[-\left(\frac{y^{\prime}}{r_{0}}+i \frac{k r_{0} y}{2 r}\right)^{2}\right]\right\} \exp \left[-\frac{k^{2} r_{0}^{2} y^{2}}{4 r^{2}}\right] \\
& \int_{-\infty}^{+\infty} d z^{\prime}\left\{\exp \left[-\left(\frac{z^{\prime}}{r_{0}}+i \frac{k r_{0} z}{2 r}\right)^{2}\right]\right\} \exp \left[-\frac{k^{2} r_{0}^{2} z^{2}}{4 r^{2}}\right]
\end{aligned}
$$

All three integrals have the same general form. Mathematica evaluates these integrals:

$$
\int_{-\infty}^{+\infty} \exp \left[-\left(\frac{\xi}{a} \pm i b\right)^{2}\right] d \xi=a \sqrt{\pi}
$$

Substituting Eq. A.8 in Eq. A.7 yields

$$
\begin{aligned}
\vec{E} & \propto \hat{z} \frac{(i \omega) r_{0}^{-3}}{c^{2} r} e^{i(k r-\omega t)}\left\{r_{0} \sqrt{\pi}\right\}^{3} \exp \left[-\frac{k^{2} r_{0}^{2}}{4}\left(1-\frac{x}{r}\right)^{2}-\frac{k^{2} r_{0}^{2} y^{2}}{4 r^{2}}-\frac{k^{2} r_{0}^{2} z^{2}}{4 r^{2}}\right] \\
& =\hat{z} \frac{(i \omega) \pi^{3 / 2}}{c^{2} r} e^{i(k r-\omega t)} \exp \left[-\frac{k^{2} r_{0}^{2}}{4}\left(\frac{x^{2}+y^{2}+z^{2}}{r^{2}}+1-2 \frac{x}{r}\right)\right]
\end{aligned}
$$


Write Eq. A.9 in spherical coordinates using $r=\sqrt{x^{2}+y^{2}+z^{2}}$ and $x=r \sin \theta \cos \phi$ :

$$
\vec{E} \propto \hat{z} \frac{(i \omega) \pi^{3 / 2}}{c^{2} r} e^{i(k r-\omega t)} \exp \left[-\frac{k^{2} r_{0}^{2}}{2}(1-\sin \theta \cos \phi)\right]
$$

To correctly identify the magnitude of the electric field, we cross $\hat{z}$ with $\hat{r}$ :

$$
\begin{aligned}
|\vec{E}| & \propto\|\hat{r} \times \hat{z}\|\left|\frac{(i \omega) \pi^{3 / 2}}{c^{2} r} e^{i(k r-\omega t)} \exp \left[-\frac{k^{2} r_{0}^{2}}{2}(1-\sin \theta \cos \phi)\right]\right| \\
& \propto|\sin \theta| e^{-k^{2} r_{0}^{2}(1-\sin \theta \cos \phi)}
\end{aligned}
$$

The electric field relates to intensity [22] through

$$
I=\frac{n c \varepsilon_{0}}{2}|\vec{E}|^{2}
$$

Inserting Eq. A.11 into Eq. A.12, we find that the electric field of the laser-driven current distribution leads to a Thomson scattering intensity distribution of

$$
I(\theta, \phi) \propto \sin ^{2} \theta e^{-k^{2} r_{0}^{2}(1-\sin \theta \cos \phi)}
$$




\section{Appendix B}

\section{Ionization Volume of a Laser Focus}

The intensity in a laser focus (example shown in Fig. B.1) as a function of position is given by

$$
\mathrm{I}=\frac{\mathrm{I}_{0}}{1+z^{2} / z_{0}^{2}} \exp \left[-\frac{2 \rho^{2}}{w_{0}^{2}\left(1+z^{2} / z_{0}^{2}\right)}\right]
$$

where $z_{0}=\pi w_{0}^{2} / \lambda$.

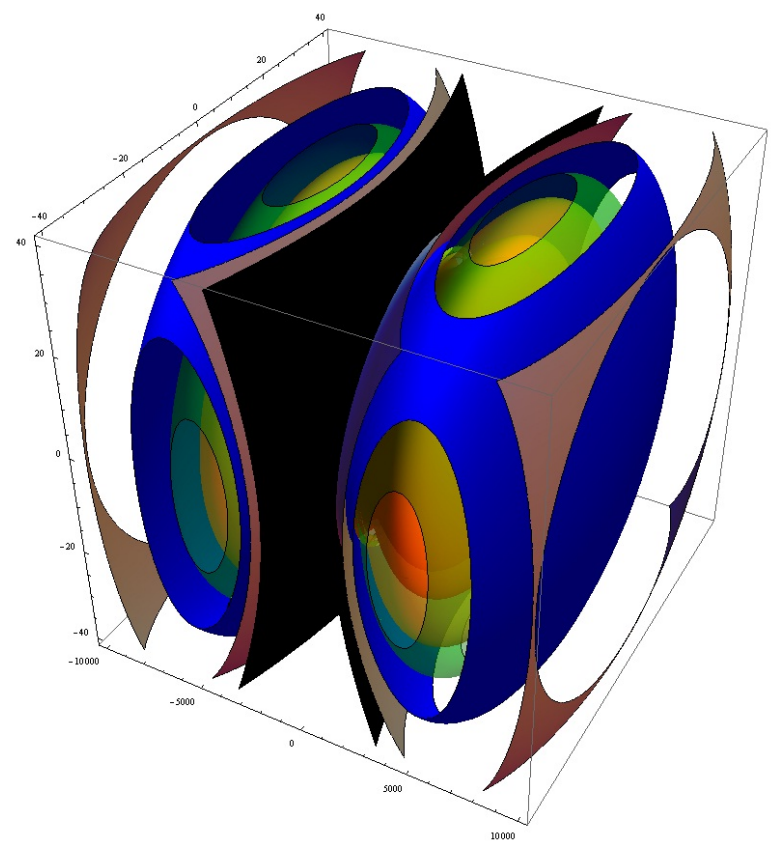

Figure B.1 Equi-intensity surfaces of a laser focus. 
In order to find the volume in the focus achieving a certain intensity, integrate using the method of disks. The differential volume element can be defined as

$$
d V=\pi \rho^{2} d z
$$

Manipulate the equation for intensity in order to find a relationship between $\rho$ and $z$.

$$
\begin{gathered}
-\frac{2 \rho^{2}}{w_{0}^{2}\left(1+z^{2} / z_{0}^{2}\right)}=\ln \left[\left(1+z^{2} / z_{0}^{2}\right) \frac{\mathrm{I}}{\mathrm{I}_{0}}\right] \\
\rho^{2}=-\frac{w_{0}^{2}\left(1+z^{2} / z_{0}^{2}\right)}{2} \ln \left[\left(1+z^{2} / z_{0}^{2}\right) \frac{\mathrm{I}}{\mathrm{I}_{0}}\right]
\end{gathered}
$$

Plug this relationship into the differential volume element, resulting in an expression completely in terms of the variable $z$ :

$$
d V=\pi\left(-\frac{w_{0}^{2}\left(1+z^{2} / z_{0}^{2}\right)}{2} \ln \left[\frac{\mathrm{I}}{\mathrm{I}_{0}}\left(1+z^{2} / z_{0}^{2}\right)\right]\right) d z
$$

Find the volume by integrating the volume element. The limits of integration are found by using the equation for intensity: by setting $\rho$ equal to 0 , solve for the extreme values of $z$ that will still satisfy the equation.

$$
\rho=0 \rightarrow z= \pm z_{0} \sqrt{\frac{\mathrm{I}_{0}}{\mathrm{I}}-1}
$$

Now integrate.

$$
\Rightarrow V=\int d V=\int \pi\left(-\frac{w_{0}^{2}\left(1+z^{2} / z_{0}^{2}\right)}{2} \ln \left[\frac{\mathrm{I}}{\mathrm{I}_{0}}\left(1+z^{2} / z_{0}^{2}\right)\right]\right) d z
$$

Mathematica shows that

$$
\int\left(a^{2}+x^{2}\right) \ln \left(a^{2}+x^{2}\right) d x=\left(\frac{x^{3}}{3}+a^{2} x\right) \ln \left(a^{2}+x^{2}\right)-\frac{2 x^{3}}{9}-\frac{4 a^{2} x}{3}+\frac{4 a^{3}}{3} \tan ^{-1} \frac{x}{a}
$$


Express the volume integral in terms of the integral from Mathematica.

$$
\Rightarrow V=\pi \sqrt{\frac{\mathrm{I}_{0}}{\mathrm{I}}}\left(-\frac{w_{0}^{2}}{2}\right) z_{0} \frac{\mathrm{I}_{0}}{\mathrm{I}} \int \frac{\mathrm{I}}{\mathrm{I}_{0}}\left(1+z^{2} / z_{0}^{2}\right) \ln \left(\frac{\mathrm{I}}{\mathrm{I}_{0}}\left(1+z^{2} / z_{0}^{2}\right)\right) d\left(\sqrt{\frac{\mathrm{I}}{\mathrm{I}_{0}}} \frac{z}{z_{0}}\right)
$$

In this case, the parameters are

$$
a=\left(\sqrt{\frac{\mathrm{I}}{\mathrm{I}_{0}}}\right), x=\left(\sqrt{\frac{\mathrm{I}}{\mathrm{I}_{0}}} \frac{z}{z_{0}}\right)
$$

Plug these specific values into the solution from Mathematica.

$$
\Rightarrow V=-\frac{\pi w_{0}^{2} z_{0}}{2}\left[\left(\frac{1}{3}\left(\frac{z}{z_{0}}\right)^{3}+\frac{z}{z_{0}}\right) \ln \left(\frac{\mathrm{I}}{\mathrm{I}_{0}}\left(1+\frac{z^{2}}{z_{0}^{2}}\right)\right)-\frac{2}{9}\left(\frac{z}{z_{0}}\right)^{3}-\frac{4}{3}\left(\frac{z}{z_{0}}\right)+\frac{4}{3} \tan ^{-1}\left(\frac{z}{z_{0}}\right)\right]
$$

Now apply the limits of the integration.

$$
\begin{array}{r}
\Rightarrow V=-\frac{\pi w_{0}^{2} z_{0}}{2}\left[-\frac{2}{9}\left(\sqrt{\frac{\mathrm{I}_{0}}{\mathrm{I}}-1}\right)^{3}-\frac{4}{3}\left(\sqrt{\frac{\mathrm{I}_{0}}{\mathrm{I}}-1}\right)+\frac{4}{3} \tan ^{-1}\left(\sqrt{\frac{\mathrm{I}_{0}}{\mathrm{I}}-1}\right)\right] \\
+\frac{\pi w_{0}^{2} z_{0}}{2}\left[\frac{2}{9}\left(\sqrt{\frac{\mathrm{I}_{0}}{\mathrm{I}}-1}\right)^{3}+\frac{4}{3}\left(\sqrt{\frac{\mathrm{I}_{0}}{\mathrm{I}}-1}\right)-\frac{4}{3} \tan ^{-1}\left(\sqrt{\frac{\mathrm{I}_{0}}{\mathrm{I}}-1}\right)\right]
\end{array}
$$

Simplify.

$$
\Rightarrow V=\pi w_{0}^{2} z_{0}\left[\frac{2}{9}\left(\sqrt{\frac{\mathrm{I}_{0}}{\mathrm{I}}-1}\right)^{3}+\frac{4}{3}\left(\sqrt{\frac{\mathrm{I}_{0}}{\mathrm{I}}-1}\right)-\frac{4}{3} \tan ^{-1}\left(\sqrt{\frac{\mathrm{I}_{0}}{\mathrm{I}}-1}\right)\right]
$$

This formula is important as we use the time-of-flight spectrometer. Since the amplitude of the oscilloscope trace is proportional to the ionization volume, it is important to know how the volumes compare to one another. Figure B.2 shows the predicted relative ionization volumes of the first eight charge states of argon. (It is worthwhile to note that the ionization volume of $\mathrm{Ar}^{8+}$ overtakes that of $\mathrm{Ar}^{7+}$ at an intensity of $5.18 \times 10^{16} \mathrm{~W} / \mathrm{cm}^{2}$. This effect is visible in Fig. 2.8.) 


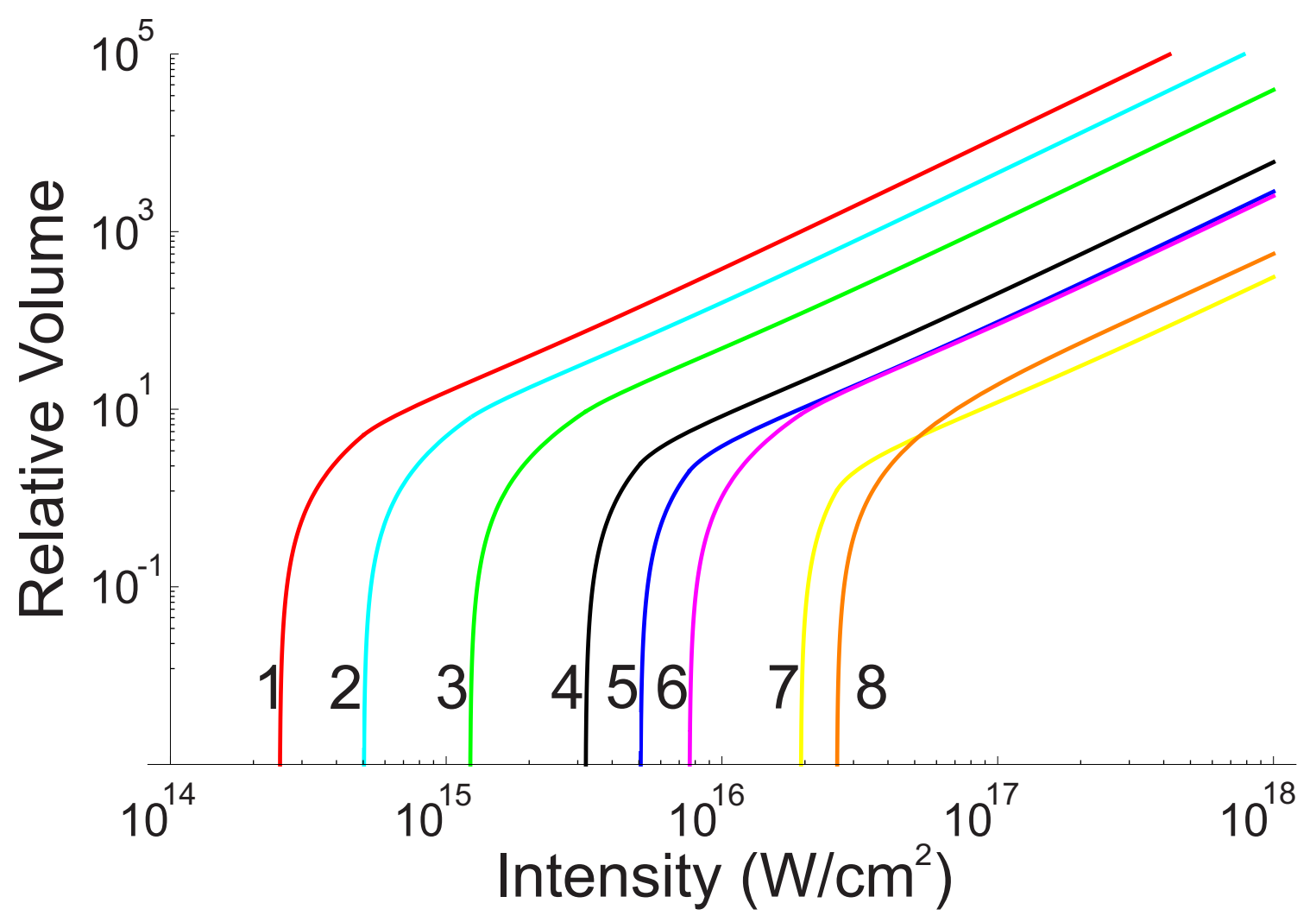

Figure B.2 Scaled ionization focal volume of argon ions as a function of intensity. Ions $\mathrm{Ar}^{+}$through $\mathrm{Ar}^{8+}$ are included.

Figure B.3 shows the predicted relative ionization volumes of the ninth and tenth charge states of argon. Because these electrons are located in a new electron shell, they emerge at much higher intensities than the previous eight electrons (as seen in Table 2.1 in Section 2.4). As a result, their ionization volumes are two orders of magnitude smaller than the next smallest ionization volume (corresponding to $\mathrm{Ar}^{7+}$ ).

It is worthwhile to note that because of the similar ionization intensities of the two electrons, the focal volumes of $\mathrm{Ar}^{9+}$ and $\mathrm{Ar}^{10+}$ are very similar in size. (The focal volume of $\mathrm{Ar}^{10+}$ overtakes that of $\mathrm{Ar}^{9+}$ at an intensity of $4.11 \times 10^{18} \mathrm{~W} / \mathrm{cm}^{2}$.) This is important because we will not be able to reach the eleventh charge state of argon with the laser (it too is located in a new electron shell). By taking the ratio between the amplitudes of $\mathrm{Ar}^{9+}$ and $\mathrm{Ar}^{10+}$ on the oscilloscope and matching 
it to a point of the theoretical ratio between the two ionization volumes, we may still be able to uniquely identify the peak intensity achieved by the laser.

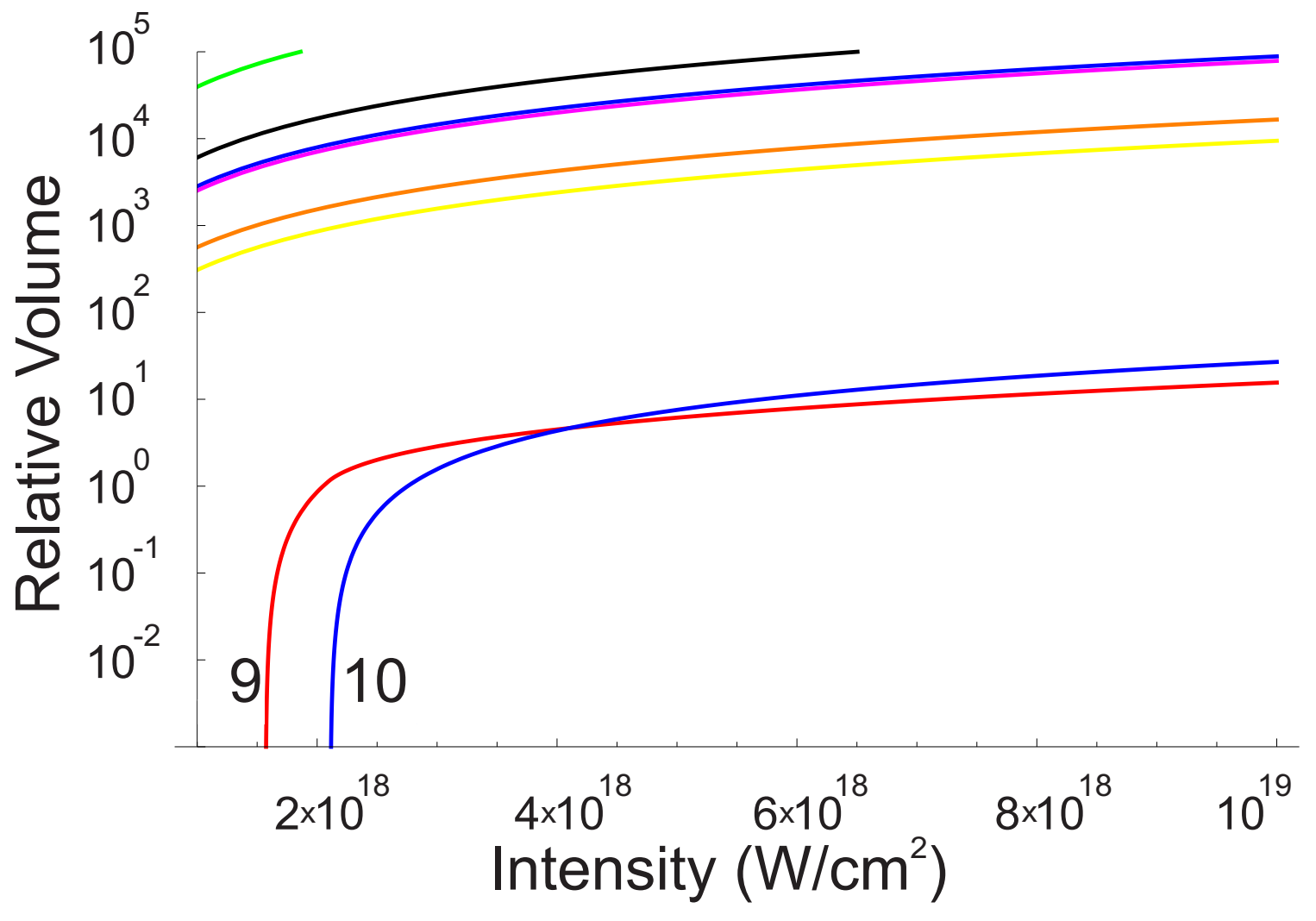

Figure B.3 Scaled ionization focal volume of argon ions as a function of intensity. Ions $\mathrm{Ar}^{9+}$ and $\mathrm{Ar}^{10+}$ are included. 



\section{Bibliography}

[1] E. A. Chowdhury, I. Ghebregziabiher, and B. C. Walker, "Larmor radiation from the ultraintense field ionization of atoms," J. Phys. B 38, 517 (2005).

[2] P. Krekora, R. E. Wagner, Q. Su, and R. Grobe, "Retardation and Kinematic Relativistic Effects in Scattered Light," Las. Phys. 12, 455-463 (2002).

[3] J. D. Jackson, Classical Electrodynamics, 3rd ed. (Wiley, New York, 1998), p. 665.

[4] J. Peatross, C. Müller, K. Z. Hatsagortsyan, and C. H. Keitel, "Photoemission of a SingleElectron Wave Packet in a Strong Laser Field," Phys. Rev. Lett. 100, 153601 (2008).

[5] J. Corson, "Photoemission of Large Electron Wave Packets," M.S. Thesis (Brigham Young University, Provo, UT, 2011).

[6] J. S. Roman, L. Roso, and H. R. Reiss, "Evolution of a relativistic wavepacket describing a free electron in a very intense laser field," J. Phys. B: At. Mol. Opt. Phys. 33, 1869-1880 (2000).

[7] J. S. Roman, L. Playa, and L. Roso, "Relativistic quantum dynamics of a localized Dirac electron driven by an intense-laser-field pulse," Phys. Rev. A 64, 063402 (2001).

[8] P. Panek, J. Z. Kaminski, and F. Ehlotzky, "Laser-induced Compton scattering at relativistically high radiation powers," Phys. Rev. A 65, 022712 (2002). 
[9] M. W. Walser, D. J. Urbach, K. Z. Hatsagortsyan, S. X. Hu, and C. H. Keitel, "Spin and radiation in intense laser fields," Phys. Rev. A 65, 043410 (2002).

[10] G. R. Mocken and C. H. Keitel, "Quantum Signatures in Laser-Driven Relativistic Multiple Scattering,” Phys. Rev. Lett. 91, 173202 (2003).

[11] J. Peatross, C. Müller, and C. H. Keitel, "Electron wave-packet dynamics in a relativistic electromagnetic field: 3-D analytical approximation,” Opt. Express 15, 6053-6061 (2007).

[12] M. Mahmoudi, Y. I. Salamin, and C. H. Keitel, "Free-electron quantum signatures in intense laser fields,” Phys. Rev. A 72, 033402 (2005).

[13] M. Verschl and C. H. Keitel, "Analytical Approach to Wave Packet Dynamics of Laser-Driven Particles beyond the Dipole Approximation," Las. Phys. 15, 529-535 (2005).

[14] G. R. Mocken and C. H. Keitel, "Quantum dynamics of relativistic electrons," J. Comput. Phys. 199, 558-588 (2004).

[15] G. R. Mocken and C. H. Keitel, "Radiation spectra of laser-driven quantum relativistic electrons," Comp. Phys. Comm. 166, 171-190 (2005).

[16] Y. I. Salamin, S. X. Hu, K. Z. Hatsagortsyan, and C. H. Keitel, "Relativistic high-power laser-matter interactions," Physics Reports 427, 41-155 (2006).

[17] K. Pangeni, "Construction of a Time-of-flight Ion Spectrometer for Calibrating Laser High Intensity," B.S. Thesis (Brigham Young University, Provo, UT, 2010).

[18] J. Peatross and S. Bergeson, "Physics 571 Lecture 33: Strong Field Ionization," http://www. physics.byu.edu/faculty/bergeson/physics571/notes/L33strongField.pdf (Accessed May 24, 2011). 
[19] R. Kretzmann, "Über optische Konstanten dicker Metallschichten im Sichtbaren und nahen Ultrarot," Ann. Physik 429, 303-325 (1940).

[20] J. Peatross and M. Ware, Physics of Light and Optics, 4th ed. (2011), p. 48.

[21] J. D. Jackson, Classical Electrodynamics, 3rd ed. (Wiley, New York, 1998), p. 247.

[22] J. Peatross and M. Ware, Physics of Light and Optics, 4th ed. (2011), p. 58. 



\section{Index}

"Double Hump" Structure, 3, 4

Aberration, 17

Avalanche Photodiode, 13, 29

Classical Current Distribution, 7

Directional Scattering Efficiency of, 8

Compton Scattering, 3

Differential Pumping, 23

Dirac Equation, 3

Electron in Relativistic Focus

Donated by Helium, 24

Point Trajectory of, 5

Wave Packet of, 12

Elliptical Mirror, 12, 18

Fiber Optic, 12, 25, 26

Gold Buffer of, 27

GRENOUILLE, 15

Ionization Volume, 21, 46

Theoretical Values of, 48

Klein-Gordon Equation, 3

Lorentz Drift, 3, 5, 6, 12

Noise Background, 7, 10-12, 15, 23, 26-29, 36

Spectral Make-up of, 38

Parametric Down-Conversion, 37

Pinhole, 12, 16, 17, 23

Ponderomotive Gradient, 5, 12

Pulse Compression, 15
Radiation

Classical Current Distribution, 8

Electron in Relativistic Focus, 5, 25

Larmor, 2

Red-shifted, 6, 10, 12, 15, 28, 36

Spectral Filtering, 12, 28

Temporal Filtering, 13, 26, 34

Thomson Scattering, 8, 43

Ti:S Laser System, 11, 13

Time Digitizer, 13, 26, 30

Time-of-Flight Spectrometer, 18, 47

XYZ Positioner, 25 